\title{
COMPLEX MULTIPLICATION CYCLES AND A CONJECTURE OF BEILINSON AND BLOCH
}

\author{
CHAD SCHOEN
}

\begin{abstract}
A generalization of the conjecture of Birch and Swinnerton-Dyer is investigated using complex multiplication cycles on a particular Kuga fiber variety. A weak finiteness result consistent with the conjecture is proved. The image of complex multiplication cycles under the Abel-Jacobi map is computed explicitly. The results provide numerical evidence supporting the conjecture. They also give evidence for a relationship between complex multiplication cycles and a modular form of weight $5 / 2$ and raise questions for further investigation.
\end{abstract}

\section{INTRODUCTION}

The purpose of this article is to subject an important conjecture concerning Chow groups to a modest but needed test. At issue is the conjecture of Beilinson $[\mathrm{Be}]$ and Bloch $[\mathrm{Bl}]$ which prèdicts for each smooth, geometrically irreducible, projective variety $V$ defined over a number field $F$ the relationship

$$
\text { rank } C H^{p}\left(V_{F}\right)_{\text {hom }}=\operatorname{ord}_{s=p} L_{F}\left(H^{2 p-1}(V), s\right) \text {. }
$$

The group $C H^{p}\left(V_{F}\right)_{\text {hom }}$ of nullhomologous, codimension $p$ algebraic cycles modulo rational equivalence is an important although often mysterious invariant of $V_{F}$. The $L$-function on the right is a holomorphic function for $\operatorname{Re}(s)>p+1 / 2$ which one hopes can be analytically continued to an entire function. Certainly an analytic continuation is necessary for $(0.1)$ to have meaning. In certain instances the analytic continuation is known to hold and the right hand side is computable. Thus the conjecture raises the distant but tempting possiblity that there is a relatively simple and occasionally computable formula for a geometric invariant which is presently intractable.

Historically (0.1) was first proposed by Birch and Swinnerton-Dyer when $p=1$ and $V$ is an elliptic curve over $\mathbb{Q}$. In the past twenty years substantial progress has been made towards verifying this special case. Our concern here is with the much more mysterious situation where the Hodge structure $H^{2 p-1}\left(V^{\text {an }}\right)$ has level $>1$. With this hypothesis there is no case for which the left-hand side of $(0.1)$ is known to be finite.

We shall work with a single projective threefold, $\widetilde{W}$, defined over $\mathbb{Q}$ and constructed from the self-fiber product of a certain elliptic modular surface

Received by the editors May 6, 1991.

1980 Mathematics Subject Classification (1985 Revision). Primary 14C25, 14G10, 11G40; Secondary $14 \mathrm{G} 35,11 \mathrm{~F} 11,11 \mathrm{~F} 37$.

Partially supported by an NSF Postdoctoral Fellowship. 
$\pi: Y \rightarrow X$ by resolving singularities. The Hodge numbers in degree 3 are $h^{3,0}(\widetilde{W})=1$ and $h^{2,1}(\widetilde{W})=0$. The $L$-function is entire and satisfies the expected functional equation. Our first main result is a finiteness theorem for a naturally defined subgroup of the Chow group of $\widetilde{W}$. To state it we note that there is a tautological morphism $p: \widetilde{W} \rightarrow X$. For each field extension $F / \mathbb{Q}$ we write $\eta_{F}$ for the generic point of $X_{F}$ and define

$$
C H^{2}\left(\widetilde{W}_{F}\right)_{\mathrm{CM}}=\operatorname{Ker}\left[C H^{2}\left(\widetilde{W}_{F}\right)_{\mathrm{hom}} \rightarrow C H^{2}\left(\widetilde{W} \times_{X} \eta_{F}\right)\right] .
$$

Theorem 0.3. If $[F: \mathbb{Q}] \leq 2$ and the sign in the functional equation for $L_{F}\left(H^{3}(\widetilde{W}), s\right)$ is positive, then $\operatorname{rank} C H^{2}\left(\widetilde{W}_{F}\right)_{\mathrm{CM}}=0$.

The rank of $C H^{2}\left(\widetilde{W}_{\mathbb{Q}}\right)_{\mathrm{CM}}$ is known to be infinite [Sch-CM]. The first part of the proof of $(0.3)$ is a geometric argument presented in $\S 2$. It is inspired by the proof that the Chow group of zero cycles for a certain surface of general type with $p_{g}=0$ and large automorphism group is finitely generated (cf. [B15, Lecture 1] and references therein). This step depends very much on the particular situation at hand and cannot be generalized even to varieties analogous to $\widetilde{W}$ which are constructed by starting with elliptic modular surfaces with larger geometric genus than $Y$. It turns out that a finite index subgroup of $C H^{2}\left(\widetilde{W}_{\mathbb{Q}}\right)_{\mathrm{CM}}$ has a very natural set of generators, so-called complex multiplication (CM) cycles, which can be described in modular terms. In particular their precise fields of definition are understood. The second step in the argument, carried out in $\S 4$, analyses the action of the Galois group on the CM cycles.

The hypothesis on the sign in the functional equation in $(0.3)$ is verified for real quadratic fields unramified at 3 . Up to a nonzero scalar factor the value of the $L$-series at the center of the critical strip is given by the Fourier coefficient $c_{d}, d=\operatorname{Disc}(F)$, in a modular form of weight $5 / 2$. Among those discriminants $d<250, d= \pm 1 \bmod 3$ only the Fourier coefficient $c_{172}$ is zero. Thus for real quadratic fields $F$ with discriminants in this range

$$
\operatorname{rank} C H^{2}\left(\widetilde{W}_{F}\right)_{\mathrm{CM}} \leq \operatorname{ord}_{s=2} L_{F}\left(H^{3}(\widetilde{W}), s\right)
$$

with equality for $d \neq 172$.

The situation which originally motivated the present work is when $F$ is an imaginary quadratic field for which $\operatorname{ord}_{s=2} L_{F}\left(H^{3}(\widetilde{W}), s\right)=1$. In this case there are infinitely many distinct $\mathrm{CM}$ cycles defined over $F$ whose image in $C H^{2}\left(\widetilde{W}_{F}\right) \otimes \mathbb{Q}$ lies in the negative eigenspace for the $\operatorname{Gal}(F / \mathbb{Q})$-action. No relations of rational equivalence among these cycles are known to the author. Nonetheless $(0.1)$ predicts that all such cycles are rationally equivalent to rational multiples of a single cycle. A consequence of this prediction will be investigated with the help of the Abel-Jacobi map from nullhomologous 1-cycles to the intermediate Jacobian. It turns out that this map can be explicitly evaluated at CM cycles $(\S 6)$. When $F=\mathbb{Q}(\sqrt{-1})$ we work through the details and with the help of a personal computer evaluate the Abel-Jacobi map for about 50 cycles. Remarkably, we find convincing numerical evidence (although no proof) that the Abel-Jacobi images are all integral multiples of a single point on the intermediate Jacobian. The integral multiples themselves are significant. As suggested by B. Gross they are, up to a fixed constant which depends only on a certain discriminant modulo 3, appropriate Fourier coefficients of the above-mentioned cusp form of weight $5 / 2$. 
An outline of the individual sections is as follows. The first section introduces the variety $\widetilde{W}$ from the three different points of view needed in the paper: As the desingularization of an explicit hypersurface in $\mathbb{P}_{\mathbb{Q}}^{2} \times \mathbb{P}_{\mathbb{Q}}^{2}$, as a modular variety, and via the uniformization of a Zariski open subset of $\widetilde{W}^{\text {an }}$. The explicit equation is then used in $\S 2$ to show that the quotients by certain modular automorphisms are rational varieties. This allows one to understand how automorphisms act on the Chow group and eventually is the basis for the proof of Theorem (0.3). The complex multiplication cycles are described in $\S 3$. The modular viewpoint leads to a precise determination of their fields of definition. The action of the Galois group on CM cycles associated to a fixed quadratic order is studied in $\S 4$. In $\S 5$ we collect basic facts concerning the $L$-function $L_{F}\left(H^{3}(\widetilde{W}), s\right)$ when $F$ is a ring class field. Root number computations suggest several themes for further investigation. One is to try to establish

$$
\operatorname{rank} C H^{2}\left(\widetilde{W}_{F}\right)_{\mathrm{CM}}=\operatorname{ord}_{s=2} L_{F}\left(H^{3}(\widetilde{W}), s\right)
$$

for many ring class fields $F$. It is a somewhat surprising fact that there is an infinite family of special ring class fields, $F$, for which $\operatorname{ord}_{s=2} L_{F}\left(H^{3}(\widetilde{W}), s\right)$ is positive and rank $C H^{2}\left(\widetilde{W}_{F}\right)_{\mathrm{CM}}=0(5.4)$. It would be interesting to "explain"the vanishing of $L_{F}\left(H^{3}(\widetilde{W}), s\right)$ in terms of zero cycles in the generic fiber of $p$.

The problem of evaluating the Abel-Jacobi map at a CM cycle is treated in $\S 6$. Here the fact that a Zariski open subset of $\widetilde{W}^{\text {an }}$ possesses a natural uniformization in terms of modular functions plays an important role. A point in the intermediate Jacobian is given by a complex number modulo a known lattice. When this point is the Abel-Jacobi image of a CM cycle, a formula for the complex number is obtained by evaluating certain power series at appropriate points in the upper half-plane (6.2). The explicit computations for cycles defined over $\mathbb{Q}(\sqrt{-1})$ are carried through in $\S 7$. In the final section the modular form of weight $5 / 2$ is discussed.

For other works which are in one way or another concerned with gathering evidence concerning the conjecture $(0.1)$ in case the Hodge structure has level greater than 1 the reader is referred to [Bl], [Bl2], [Br], [Ha], [Top], and [Ze].

Acknowledgments. I wish to thank B. Gross and S. Bloch for help and encouragement. In particular, I am indebted to Gross for the suggestion that the integers appearing in Table 7.5 might be related to Fourier coefficients of a modular form of half-integral weight. As it turned out the relevant modular form had previously been introduced by N. Koblitz. I thank him for making me aware of his work. The verification that the derivative of the $L$-function in (8.4) does not vanish at $s=2$ was furnished by Bloch. I am grateful for the hospitality of the IAS (1982-83) and MSRI (1986-87) where parts of this work were done and for the support of the NSF.

Notations. $\quad D=$ discriminant of an order in an imaginary quadratic number field.

$$
\begin{aligned}
& \qquad \mathscr{O}_{D}=\mathbb{Z}[(D+\sqrt{D}) / 2]=\text { the order of discriminant } D \\
& k_{D}=\mathbb{Q}(\sqrt{D}) \\
& H_{D}=k_{D}\left(j\left(\mathscr{O}_{D}\right)\right) \text {, where } j\left(\mathscr{O}_{D}\right) \text { denotes the classical modular function eval- } \\
& \text { uated at the lattice } \mathscr{O}_{D} \text {. }
\end{aligned}
$$


$E[n]=$ the $n$-torsion points on an elliptic curve $E$.

$\langle s\rangle=$ the cyclic subgroup generated by an element $s$.

$\mu_{n}=\left\langle\zeta_{n}\right\rangle=$ the group of $n$ th-roots of unity.

$K=\mathbb{Q}\left(\mu_{3}\right)$.

$\mathbf{h}=$ the complex upper half-plane.

Given an $n$-dimensional variety $V$ over a subfield $F \subset \mathbb{C}$ define $V^{\text {an }}=$ the complex analytic space associated to $V$.

$Z^{p}\left(V_{F}\right)=Z_{n-p}\left(V_{F}\right)=$ the group of codimension $p$ algebraic cycles on $V_{F}$. $Z^{p}\left(V_{F}\right)_{\text {rat }}=$ the subgroup of cycles which are rationally equivalent to zero. $Z^{p}\left(V_{F}\right)_{\text {hom }}=$ the kernel of the cycle class map cl $: Z^{p}\left(V_{F}\right) \rightarrow H^{2 p}\left(V^{\text {an }}, \mathbb{Z}\right)$. $C H^{p}\left(V_{F}\right)=Z^{p}\left(V_{F}\right) / Z^{p}\left(V_{F}\right)_{\text {rat }}$.

$C H^{p}\left(V_{F}\right)_{\text {hom }}=Z^{p}\left(V_{F}\right)_{\text {hom }} / Z^{p}\left(V_{F}\right)_{\text {rat }}$.

\section{THE MODULAR VARIETY}

This section collects basic facts about the elliptic modular threefold $\widetilde{W}$ which is the focus of this paper. The reader may find a more leisurely exposition of certain points treated quickly here in [Shd] and [Sch-CM].

(1.1) The variety $\widetilde{W} / \mathbb{Q}$ will be constructed from the smooth rational surface $Y \subset \mathbb{P}_{\mathbb{Q}}^{1} \times \mathbb{P}_{\mathbb{Q}}^{2}$ defined by the bihomogeneous equation

$$
u_{0}\left(x_{0}^{3}+x_{1}^{3}+x_{2}^{3}\right)-u_{1} x_{0} x_{1} x_{2}=0 .
$$

Projection onto the first factor, which shall be denoted $\pi: Y \rightarrow X$, gives rise to the structure of an elliptic surface on $Y$. The remarkable feature of the corresponding elliptic pencil on $\mathbb{P}^{2}$ is that each of the nine base points is an inflection point for every curve in the family. Thus when $e=(0:-1: 1)$ is taken to be the origin, the remaining base points account for all the three torsion. The singular fibers of $\pi$ are of Kodaira type $I_{3}$ and lie over the cusps $\left\{\infty, 3 \mu_{3}\right\} \subset \mathbb{P}^{1}$, where $u_{1} / u_{0}$ is taken to be the inhomogeneous coordinate on $\mathbb{P}^{1}$. Removing these points gives rise to an abelian scheme $\dot{\pi}: \dot{Y} \rightarrow \dot{X}:=$ $\mathbb{P}^{1}-\left\{\infty, 3 \mu_{3}\right\} \simeq$ Spec $\mathbb{Q}\left[u,\left(u^{3}-27\right)^{-1}\right]$.

To define $\widetilde{W}$, start by letting $W:=Y \times_{\pi} Y$, and $\dot{W}:=\dot{Y} \times_{i} \dot{Y}$. Then $\tilde{\sigma}: \widetilde{W} \rightarrow W$ will be the blow up of $W$ along $W_{\text {sing }}$ which consists of ordinary double points. These occur at points $\left(v_{1}, v_{2}\right)$ where $v_{1}, v_{2} \in \pi^{-1}(x)$ are both singular points of the fiber. Write $p: W \rightarrow X$ for the obvious map and $\dot{p}=\left.p\right|_{\dot{W}}, \tilde{p}=p \circ \tilde{\sigma}$. Evidently $W$ is the hypersurface in $Y \times Y$ which under the natural morphism $Y \times Y \rightarrow \mathbb{P}^{2} \times \mathbb{P}^{2}$ maps birationally to the hypersurface, $\bar{W}$,

$$
x_{0} x_{1} x_{2}\left(y_{0}^{3}+y_{1}^{3}+y_{2}^{3}\right)=y_{0} y_{1} y_{2}\left(x_{0}^{3}+x_{1}^{3}+x_{2}^{3}\right) .
$$

(1.2) It is important to give an interpretation of these varieties in the context of moduli of elliptic curves with level structure. The appropriate notion for the case at hand will be refered to as a weak level-3-structure. By such a structure on an abelian scheme $E$ of relative dimension 1 over a scheme $S$, we understand a section $s_{1}$ of exact order 3 together with a subgroup scheme $\mathscr{H} \subset E$ which is isomorphic to $\mu_{3}$ over $S$ and which is disjoint from $s_{1}$. Setting $s_{1}=(-1: 0: 1)$ and $s_{2}=\left(0:-\zeta_{3}: \zeta_{3}^{2}\right)$ gives rise to the weak level 3-structure $\left(s_{1},\left\langle s_{2}\right\rangle\right)$ on $\dot{Y}$. In fact $\left(\dot{Y}, s_{1},\left\langle s_{2}\right\rangle\right)$ is the universal family which represents the functor, $F$ : Affine $\mathbb{Q}$-algebras without zero-divisors $\rightarrow$ Sets, 
defined by $F(A)=$ isomorphism classes of abelian schemes of relative dimension 1 with weak level-3-structure over $\operatorname{Spec} A$. This is a remarkable property in the sense that the universal families for most similar moduli problems cannot be described by such simple equations. The connection between $\dot{\pi}$ and the moduli of elliptic curves was realized in the last century (cf. [Bi]). The precise statement that $\dot{\pi}$ represents $F$ can be established with relatively little machinery. A few sentences will suffice to roughly sketch the idea. The main point is that given $\left(E, s_{1}, \mathscr{H}\right)$ over Spec $A$ one can explicitly describe the induced map Spec $A \rightarrow$ Spec $\mathbb{Q}\left[u,\left(u^{3}-27\right)^{-1}\right]$ as follows: The Cartier divisor $\left(\mathscr{H}+s_{1}\right)-(\mathscr{C})$ on $E$ gives rise to an invertible sheaf $L$ such that $\left.L\right|_{\left[2 s_{1}\right]}$ has a nowhere vanishing section. As $L$ has trivial restriction to each fiber, it is the pullback of an invertible sheaf on Spec $A$ [H, III Ex. 12.4], whence $L \simeq O_{E}$. There is a unique rational function $t_{1}$ on $E$ which is identically -1 along the section $\left[2 s_{1}\right]$ and has divisor $\left(t_{1}\right)=\left(\mathscr{H}+s_{1}\right)-(\mathscr{H})$. Write $t_{2}$ for the transform of $t_{1}$ by inversion on $E$. It may be shown that the rational function $\mu=\left(1+t_{1}^{3}+t_{2}^{3}\right) / t_{1} t_{2}$ has trivial divisor on geometric fibers. In fact after adjoining a cube root of unity to $A$, we may choose a generator $s_{2}$ of $\mathscr{H}$. Let $\mathbf{s}_{i}$ denote translation by the section $s_{i}$. The divisor of $\left(1+t_{1}+t_{2}\right)$ is $\left[s_{1}\right]+\left[2 s_{1}\right]-\left[s_{2}\right]-\left[2 s_{2}\right]$, whence

$$
g=\left(\prod_{1 \leq i \leq 3}\left(\mathbf{s}_{2}^{i}\right)^{*}\left(1+t_{1}+t_{2}\right)\right) / t_{1} t_{2}
$$

has trivial divisor. But $\mathbf{s}_{2}^{*} t_{i}=\zeta_{3}^{i} t_{i}$, which implies $\mu=g+3$. Thus $\mu$ is pulled back from $A$. The map $f: \mathbb{Q}[u] \rightarrow A, f(u)=\mu$ lifts to $\mathbb{Q}\left[u,\left(u^{3}-27\right)^{-1}\right]$. The task of showing that this map of functors

$$
F \rightarrow \operatorname{Morph}\left(, \operatorname{Spec} \mathbb{Q}\left[u,\left(u^{3}-27\right)^{-1}\right]\right)
$$

is a isomorphism is left to the reader (cf. [M-S]).

(1.3) Our approach to the Abel-Jacobi map for 1-cycles on $\widetilde{W}$ is based on the fact that the analytic space $\dot{Y}^{\text {an }}$ and hence $\dot{W}^{\text {an }}$ has a natural uniformization given by modular functions. To describe this let $\Gamma(3):=\operatorname{Ker} S L(2, \mathbb{Z}) \rightarrow$ $S L(2, \mathbb{Z} / 3)$ and let $\widetilde{\Gamma}(3)_{j}$ denote the semidirect product $\Gamma(3) \cdot\left(\mathbb{Z}^{j}\right)^{2}$ with multiplication

$$
(\alpha,(m, n)) *\left(\beta,\left(m^{\prime}, n^{\prime}\right)\right)=\left(\alpha \beta,(m, n) \beta+\left(m^{\prime}, n^{\prime}\right)\right),
$$

where $m, n, m^{\prime}, n^{\prime} \in \mathbb{Z}^{j}$ are column vectors. Let $\mathrm{h}$ denote the upper halfplane. There is a natural free action of $\widetilde{\Gamma}(3)_{j}$ on $\mathbf{h} \times \mathbb{C}^{j}$ given by

$$
\begin{aligned}
\left(\left(\begin{array}{ll}
a & b \\
c & d
\end{array}\right),(m, n)\right) *(\tau, z) \\
\quad=\left((a \tau+b) /(c \tau+d),(c \tau+d)^{-1}(z+\tau m+n)\right) .
\end{aligned}
$$

The quotient $\widetilde{\Gamma}(3)_{1} \backslash \mathbf{h} \times \mathbb{C}$ is a family of genus 1 Riemann surfaces with sections $s_{i}: \Gamma(3) \backslash \mathbf{h} \rightarrow \widetilde{\Gamma}(3)_{1} \backslash \mathbf{h} \times \mathbb{C}, i \in\{0,1,2\}$, given by $s_{0}(\tau)=(\tau, 0), s_{1}(\tau)=$ $(\tau, 1 / 3), s_{2}(\tau)=(\tau, \tau / 3)$. Take $s_{0}$ to be the zero section so that $s_{1}$ and $s_{2}$ have exact order 3. Meromorphic functions $t_{1}, t_{2}$ yielding a map $\widetilde{\Gamma}(3)_{1} \backslash \mathbf{h} \times \mathbb{C} \rightarrow$ $\dot{Y}^{\text {an }}$ may be constructed with the help of the Weierstrass sigma function:

$$
\sigma(\tau, z)=z \prod_{(a, b) \neq(0,0)}(1-z /(a \tau+b)) e^{z / a \tau+b} e^{(z / a \tau+b)^{2} / 2} .
$$


Define

$$
\begin{aligned}
& x_{0}(\tau, z)=\sigma(\tau, z) \sigma(\tau, z-\tau / 3) \sigma(\tau, z+\tau / 3) \\
& x_{1}(\tau, z)=\sigma(\tau, z+2 / 3) \sigma(\tau, z-1 / 3-\tau / 3) \sigma(\tau, z-1 / 3+\tau / 3) \epsilon(\tau), \\
& x_{2}(\tau, z)=\sigma(\tau, z-2 / 3) \sigma(\tau, z+1 / 3-\tau / 3) \sigma(\tau, z+1 / 3+\tau / 3) \epsilon(\tau),
\end{aligned}
$$

where

$$
\epsilon(\tau)=-\sigma(\tau, 1 / 6) / \sigma(\tau,-5 / 6)
$$

Now set $t_{1}=x_{1} / x_{0}, t_{2}=x_{2} / x_{0}$. Using the transformation properties of the $\sigma$ function $[\mathrm{La}, \S 18]$ one can verify

Lemma 1.4. (1) The functions $t_{1}, t_{2}$ on $\mathbf{h} \times \mathbb{C}$ are invariant under the action of $\widetilde{\Gamma}_{1}(3)$.

(2) $t_{1}(\tau, 2 / 3)=-1$.

(3) $t_{2}(\tau, z)=t_{1}(\tau,-z)$.

(4) $\overline{t_{i}(\tau, z)}=t_{i}(-\bar{\tau}, \bar{z})$.

By (1), (2), (3) the situation is analogous to (1.2). In particular, the function $\mu(\tau, z)=\left(1+t_{1}^{3}+t_{2}^{3}\right) / t_{1} t_{2}$ is independent of $z$. There is a commutative diagram of holomorphic maps

$$
\begin{array}{ccc}
\widetilde{\Gamma}(3)_{1} \backslash \mathbf{h} \times \mathbb{C} \stackrel{(1: \mu)\left(1: t_{1}: t_{2}\right)}{\longrightarrow} & \dot{Y}^{\text {an }} \\
\mathrm{pr}_{\mathbf{h}} \downarrow & \downarrow \pi \\
\Gamma(3) \backslash \mathbf{h} & \stackrel{(1: \mu)}{\longrightarrow} & \mathbb{P}_{\mathbb{C}}^{1}-\left\{\infty, 3 \mu_{3}\right\},
\end{array}
$$

which is an isomorphism on fibers. It is bijective on base curves, as each elliptic curve with weak level-3-structure occurs exactly once in each family. It follows that the top horizontal arrow is an isomorphism. The theory of torus embeddings gives rise to a natural compactification $B$ of $\widetilde{\Gamma}(3)_{1} \backslash \mathbf{h} \times \mathbb{C}$ [AMRT, I.4]. The above map then extends to an isomorphism $B \rightarrow Y^{\text {an }}$.

(1.5) For the numerical computation of the Abel-Jacobi map, it is crucial to have a manageable basis for $F^{2} H^{3}(\widetilde{W}, \mathbb{C})$. Such is provided by the classical theory of modular forms. Indeed, the transformation formula for the Dedekind eta function, $\eta(\tau)=\exp (2 \pi i \tau / 24) \prod_{n \geq 1}(1-\exp (2 \pi i n \tau))$ [La, p. 259], shows that $\omega=(2 \pi i / 3) \eta^{8}(\tau) d \tau d z_{1} d z_{2}$ is $\widetilde{\Gamma}_{2}(3)$-invariant, hence descends to a global holomorphic 3-form on $\dot{W}^{\text {an }}$. Using the explicit description of the compactification $B$ of $\dot{Y}^{\text {an }}$ [AMRT, I.4], one checks that $\omega$ extends to a holomophic form on $W^{\text {an }}-W_{\text {sing }}$, and hence to a global section of the dualizing sheaf $\omega_{W}$. It follows that $\omega$ yields a global holomorphic 3-form on $\widetilde{W}^{\text {an }}$. In fact $\omega \in F^{2} H^{3}\left(\widetilde{W}^{\text {an }}, \mathbb{C}\right)$ is a basis as the next lemma shows.

Lemma 1.6. The numerical invariants of $\widetilde{W}^{\text {an }}$ are as follows.
(1) $\pi_{1}\left(\widetilde{W}^{\text {an }}\right) \simeq 1$
(2) $h^{2,0}\left(\widetilde{W}^{\text {an }}\right)=0$
(3) $h^{2,1}\left(\widetilde{W}^{\text {an }}\right)=0$
(4) $\quad \omega_{W} \simeq \mathscr{O}_{W}, h^{0}\left(\widetilde{W}, \omega_{\widetilde{W}}\right)=1$. 
Proof. (1) [Sch-CM, 1.1], (2) [Sch-CM, 1.2], (3) [Sch-CM, 1.7], (4) [Sch-FP, $\S 7]$.

\section{THE ACTION OF AUTOMORPHISMS ON THE CHOW GROUP}

In this section we describe how certain obvious automorphisms of $\widetilde{W}_{\mathbb{C}}$ act on $C H^{2}\left(\widetilde{W}_{\mathbf{C}}\right)_{\text {hom }} \otimes \mathbb{Q}$. These automorphisms arise naturally from automorphisms of the corresponding moduli problem which we now describe.

(2.1) To begin fix a primitive cube root of unity $\zeta_{3}$. Given an elliptic curve with weak level-3-structure $\left(E, s_{1}, \mathscr{H}\right)$ defined over an extension field of $\mathbb{Q}\left(\zeta_{3}\right)$, let $s_{2} \in \mathscr{H}$ denote the unique generator for which the Weil pairing yields $\left\langle s_{1}, s_{2}\right\rangle=\zeta_{3}$. Thus if $\zeta_{3}$ is in the base field, a weak level-3-structure is the same as a symplectic level-3-structure. In particular $Y_{\mathbb{Q}\left(\zeta_{3}\right)}$ is the moduli space for elliptic curves with symplectic level-3-structure. Changing the choice of symplectic level 3-structure gives rise to an action of $S L(2, \mathbb{Z} / 3)$ on $Y_{\mathbb{Q}\left(\mu_{3}\right)}$. Only the automorphisms \pm Id are defined over $\mathbb{Q}$, although in the induced action of $S L(2, \mathbb{Z} / 3) / \pm$ Id on the modular curve $X_{\mathbb{Q}\left(\mu_{3}\right)}$ elements of the Sylow2 -subgroup are defined over $\mathbb{Q}$. Explicit generators in terms of the coordinates introduced in (1.2) are given by $U_{1}$ and $S_{1}$, where $x_{0} \circ U_{1}=\zeta_{3} x_{0}, x_{i} \circ$ $U_{1}=x_{i}, i \in\{1,2\}, u_{0} \circ U_{1}=\zeta_{3} u_{0}, u_{1} \circ U_{1}=u_{1}$ and $S_{1}$ acts on the right on the $x$-coordinates by the matrix $\left(\left(\zeta_{3}\right)^{i j}\right)_{0 \leq i, j \leq 2}$ and on $u$-coordinates by $\left(u_{0}: u_{1}\right) \circ S_{1}=\left(-3 u_{0}+u_{1}: 18 u_{0}+3 u_{1}\right)$. In terms of the uniformization of $\dot{Y}^{\text {an }}$, the $S L(2, \mathbb{Z} / 3)$-action is induced by the obvious action of the semidirect product $S L(2, \mathbb{Z}) \cdot\left(\mathbb{Z}^{2}\right)(1.3 .1)$. Now let $S L(2, \mathbb{Z} / 3)$ act diagonally on $W$ and hence on $\widetilde{W}$. By the product formula for $\eta^{8}(\tau)$, the matrix $\left(\begin{array}{ll}1 & 1 \\ 0 & 1\end{array}\right)$ transforms this function by a primitive cube root of unity. Thus the action of $S L(2, \mathbb{Z} / 3)$ on the one dimensional vector space $H^{0}\left(\widetilde{W}, \omega_{\widetilde{W}}\right)$ is nontrivial, and in fact necessarily factors through the quotient group $\mathbb{Z} / 3$.

(2.2) Write $M$ for the unique nontrivial irreducible $\mathbb{Q}[\mathbb{Z} / 3]$-module viewed as a module over $\mathbb{Q}[S L(2, \mathbb{Z} / 3)]$ via the obvious homomorphism $\mathbb{Q}[S L(2, \mathbb{Z} / 3)] \rightarrow \mathbb{Q}[\mathbb{Z} / 3]$.

Proposition 2.2.1. $C H^{2}\left(\widetilde{W}_{\mathbb{C}}\right)_{\text {hom }} \otimes \mathbb{Q}$ is isomorphic to a direct sum of simple $\mathbb{Q}[S L(2, \mathbb{Z} / 3)]$-modules $M$.

We begin with the observation

Lemma 2.2.2. - Id $\in S L(2, \mathbb{Z} / 3)$ acts trivially on $C H^{2}\left(\widetilde{W}_{\mathbb{C}}\right)_{\text {hom }} \otimes \mathbb{Q}$.

Proof. Write $\eta_{\mathbb{C}}$ for the generic point of $X_{\mathbb{C}}$. Tensoring the localization sequence with $\mathbb{Q}$ gives an exact sequence

$$
\bigoplus_{x \in X(\mathbf{C})} C H^{1}\left(p^{-1}(x)\right) \otimes \mathbb{Q} \stackrel{\oplus \psi_{x}}{\longrightarrow} C H^{2}\left(\widetilde{W}_{\mathbb{C}}\right) \otimes \mathbb{Q} \rightarrow C H^{2}\left(p^{-1}\left(\eta_{\mathbb{C}}\right)\right) \otimes \mathbb{Q} \rightarrow 0 .
$$

Since

$$
\operatorname{Alb}_{p^{-1}\left(\eta_{\mathrm{c}}\right)}(\mathbb{C}(X)) \simeq \operatorname{Pic}_{p^{-1}\left(\eta_{\mathrm{c}}\right)}^{0}(\mathbb{C}(X)) \simeq\left(\mathrm{Pic}_{\pi^{-1}\left(\eta_{\mathrm{c}}\right)}^{0}(C(X))\right)^{2} \simeq(\mathbb{Z} / 3)^{4},
$$

the image of $C H^{2}\left(\widetilde{W}_{\mathbf{C}}\right)_{\text {hom }} \otimes \mathbb{Q}$ in $C H^{2}\left(p^{-1}\left(\eta_{\mathbb{C}}\right)\right)_{\text {hom }} \otimes \mathbb{Q}$ lies in the kernel of the Albanese map. Inversion on the abelian variety induces the identity map on this group of cycle classes [B13, A.9]. 
Since $W$ is the fiber product of rational surfaces, cycles which are algebraically equivalent to zero in a smooth, closed fiber $p^{-1}(x)$ are rationally equivalent to zero on $W$. Thus $\psi_{x}: C H^{1}\left(p^{-1}(x)\right) \rightarrow C H^{2}(\widetilde{W})$ factors through the Neron-Severi group, $\mathrm{NS}\left(p^{-1}(x)\right)$. Inversion on $p^{-1}(x)$ induces the identity on $\mathrm{NS}\left(p^{-1}(x)\right)$. Thus a nullhomologous cycle $z \in \operatorname{im}\left(\bigoplus_{x \in X(C)} \psi_{x}\right)$ is rationally equivalent to a nullhomologous cycle, $z_{0}$, supported on the singular fibers plus cycle classes on which - Id acts trivially. However a multiple of $z_{0}$ is rationally equivalent to zero [Sch-CM, 5.3]. The lemma follows.

The main step in the proof of $(2.2 .1)$ is the

Lemma 2.2.3. Given any $U \in S L(2, \mathbb{Z} / 3)$ of order 3 , the quotient $\langle U\rangle \backslash W_{\mathbb{C}}$ is a rational variety with a smooth model $V$ for which $H^{3}(V, \mathbb{Q})=0$

Proof. As all subgroups of order 3 in $S L(2, \mathbb{Z} / 3)$ are conjugate it suffices to check the assertions for any particular order three element. Consider the model $\bar{W} \subset \mathbb{P}^{2} \times \mathbb{P}^{2}(1.1)$ and the order three automorphism $U$ given by $U_{1}$ acting diagonally. Pass to the affine coordinate system $\mathbf{x}_{i}=x_{i} / x_{2}, \mathbf{y}_{i}=y_{i} / y_{2}$ and then make the substitutions $c=\mathbf{y}_{0} / \mathbf{x}_{0}, e=\mathbf{y}_{1} / \mathbf{x}_{1}$ so that the equation for $\bar{W}$ becomes

$$
\mathbf{y}_{0}^{3}\left(c^{3}-c e\right)+\mathbf{y}_{1}^{3}\left(e^{3}-c e\right)+1-c e=0 .
$$

Since $\mathbf{y}_{1}, c, e$ are fixed by $U$ and $\mathbf{y}_{0} \circ U=\zeta_{3} \cdot \mathbf{y}_{0}$ the rationality of the quotient is clear.

The fixed point set for the action of $U_{1}$ on $Y$ consists of the line $x_{0}=0$ and the point $x_{1}=x_{2}=0$ in the fiber $\pi^{-1}(\infty)$ together with some isolated fixed points in the smooth fiber $\pi^{-1}(0)$. Thus the fixed point locus of $U$ acting on $\widetilde{W}_{\mathrm{C}}$ is a disjoint union of isolated points, smooth rational curves and smooth divisors. A local computation, using the fact that $U$ has order three, shows that there is a composition of blow ups $\breve{W} \rightarrow \widetilde{W}_{\mathbb{C}}$ centered at isolated points and along smooth rational curves with the property that $U$ acts on $\check{W}$ with nonsingular quotient $V$. Since $H^{3}\left(\widetilde{W}^{\text {an }}, \mathbb{Q}\right) \simeq H^{3}\left(\check{W}^{\text {an }}, \mathbb{Q}\right)$ and there are no $U$-invariants in $H^{3}\left(\widetilde{W^{a}}, \mathbb{Q}\right)$, we have $H^{3}(V, \mathbb{Q})=0$.

Corollary 2.2.4. If $U \in S L(2, \mathbb{Z} / 3)$ has order 3 , then $\left(1+U+U^{2}\right) \cdot C H^{2}\left(\widetilde{W}_{\mathbb{C}}\right)_{\mathrm{hom}}$ $=0$.

Proof. By the moving lemma, a class in $C H^{2}\left(\widetilde{W}_{\mathbb{C}}\right)_{\text {hom }}$ may be represented by a cycle $z$ whose support is disjoint from the fundamental locus of the correspondence $\varrho: \widetilde{W} \rightarrow V$. Clearly

$$
z+U z+U^{2} z=\varrho^{*} \circ \varrho_{*}(z) .
$$

For a nonsingular rational 3-fold, $V / \mathbb{C}, C H^{2}(V)_{\text {hom }}$ is isomorphic to Murre's abelian variety $\left[\mathrm{Bl}-\mathrm{S}\right.$, Theorem 1], which is zero when $H^{3}(V, \mathbb{Q})=0[\mathrm{Mu}]$.

It is now apparent that $C H^{2}\left(\widetilde{W}_{\mathbb{C}}\right)_{\text {hom }} \otimes \mathbb{Q}$ is a direct sum of the simple $\mathbb{Q}[\operatorname{PSL}(2, \mathbb{Z} / 3)]$-modules with the property that for each order 3 element $U$, $\left(1+U+U^{2}\right)$ annihilates the module. There are three isomorphism classes of simple modules over this group ring, namely $M$, the trivial module, and the Steinberg module. The latter has dimension 3 over $\mathbb{Q}$. Of these, only $M$ is annihilated by $\left(1+U+U^{2}\right)$. 
(2.3) It would be interesting to know if the representation of the algebra generated by Hecke correspondences on $C H^{2}\left(\widetilde{W}_{\mathbb{C}}\right)_{\text {hom }} \otimes \mathbb{Q}$ also has a simple description.

(2.4) The analog of (2.2.2) for those elliptic modular threefolds $\widetilde{W}(\Gamma)$ [Sch$\mathrm{CM}, \S 1]$ for which $h^{2,0}(\widetilde{W}(\Gamma))$ is nonzero is false when the base field is the complex numbers. This follows from the fact that the Chow group of zero cycles on the elliptic modular surface $Y(\Gamma)$ is large and that - Id acts as -1 on $\operatorname{Pic}^{0}\left(p^{-1}(x)\right)$ [Sch-CM, p.780].

\section{PReliminaries on COMPLEX MUltiplication CyCles}

(3.1) The variety $\widetilde{W}_{\mathbb{Q}}$ is equipped with a remarkable collection of nullhomologous 1-cycles supported on those fibers $p^{-1}(x)$ where the Picard number jumps. We recall the definition of these so-called complex multiplication cycles from [Sch-CM, §2].

Suppose given an elliptic curve $E$ defined over a number field $L$ with the property that $\operatorname{End}\left(E_{L}\right)$ is isomorphic to the unique quadratic order, $\mathscr{O}_{D}$, of some discriminant $D<0$. The complex multiplication cycle $z_{E}$ is the positive generator of the free, rank one, oriented subgroup of $\operatorname{NS}(E \times E)$ which is orthogonal to $\operatorname{Span}\{E \times e, e \times E, \Delta\}$. The orientation is specified with the help of the standard map [Sch-CM, p. 799]

$$
\phi: \mathrm{NS}(E \times E) \rightarrow \operatorname{End}\left(C H_{0}(E)_{\operatorname{deg} 0}\right)=\operatorname{End}\left(E_{L}\right)
$$

which sends $\operatorname{Span}\{E \times e, e \times E, \Delta\}$ to $\mathbb{Z}$. It is determined by choosing a positive half-space $\operatorname{End}\left(E_{L}\right)^{+} \subset \operatorname{End}\left(E_{L}\right)$. This is done systematically by the following conventions: First, all number fields in this paper are assumed to be subfields of $\mathbb{C}$. A choice of $\sqrt{-1}$, and hence of an upper half-space in $\mathbf{h} \subset \mathbb{C}$ is made once and for all. Secondly, we embed $\operatorname{End}\left(E_{L}\right)$ in $L$ by means of the action of endomorphisms on the rank one $L$-module $H^{0}\left(E, \Omega_{E / L}\right)$. Set $\operatorname{End}\left(E_{L}\right)^{+}=\operatorname{End}\left(E_{L}\right) \cap \mathbf{h}$. If $\nu \in \operatorname{End}\left(E_{L}\right)$ is in the positive half-space and $\mathbb{Z}[\nu]=\operatorname{End}\left(E_{L}\right)$, then $z_{E}$ can be written in terms of the graphs of the endomorphisms $\nu$ and $\bar{\nu}$,

$$
z_{E}=(\epsilon(D) / 2) \cdot\left(\Gamma_{\nu}-\Gamma_{\bar{\nu}}\right)
$$

where $\epsilon(D)=1$ (respectively 2) when $D$ is even (respectively odd). B. Gross points out that $z_{E}$ is the unique element of $\operatorname{Span}\{E \times e, e \times E, \Delta\}^{\perp}$ such that $\phi\left(z_{E}\right)$ is the positive trace zero element in $\operatorname{End}\left(E_{L}\right)$ of minimal norm. Other characterizations of this cycle are given in [Sch-CM, 2.4].

(3.2) The set $C M_{D}=\left\{x \in \dot{X}(\mathbb{Q}): \operatorname{End}\left(\pi^{-1}(x)\right)=\mathscr{O}_{D}\right\}$ will be called the set of $C M$ points of discriminant $D$. For $x \in C M_{D}$, set $E=\pi^{-1}(x)$ and let $z_{x} \in C H_{1}\left(\widetilde{W}_{\mathbb{Q}}\right)$ denote the image of $z_{E}$ under the homomorphism $\psi_{x}$ : $\mathrm{NS}(E \times E) \rightarrow C H_{1}\left(\widetilde{W}_{\mathbb{Q}}\right)$ induced by inclusion. Recall from the proof of $(2.2 .2)$ that $\psi_{x}$ is well defined. By [Sch-CM, 2.5], the complex multiplication cycle, $z_{x}$, is homologous to zero in $\widetilde{W}_{\mathbb{Q}}$.

Lemma 3.2.1. Complex multiplication cycles generate a finite index subgroup of

$$
\operatorname{Ker}\left[C H^{2}\left(W_{\mathbb{Q}}\right)_{\text {hom }} \rightarrow C H^{2}\left(W \times_{X} \eta_{\mathbb{Q}}\right)\right] .
$$


Proof. Clearly all complex multiplication cycles are contained in the kernel. The divisors $\pi^{-1}(x) \times e, e \times \pi^{-1}(x)$, and $\Delta$ on a smooth fiber $p^{-1}(x)$ are restrictions of obvious divisors $D_{1}, D_{2}, D_{3}$ on $\widetilde{W}$. By intersecting with $D_{1}, D_{2}, D_{3}$ one sees that for $\left.\sum_{x} \sum_{1 \leq i \leq 3} a_{x}^{i} D_{i}\right|_{p^{-1}(x)}$ to be homologous to zero on $\widetilde{W}_{\mathbb{Q}}$ it is necessary that $\sum_{x} a_{x}^{i}=0$ for each $i$. As the base curve $X$ is $\mathbb{P}^{1}$, this implies that $\left.\sum_{x} a_{x}^{i} D_{i}\right|_{p^{-1}(x)}$ is rationally equivalent to zero. Thus a nullhomologous 1-cycle on $\widetilde{W}_{\mathbb{Q}}$ supported on closed fibers is rationally equivalent to a linear combination of complex multiplication cycles and a cycle supported on the singular fibers. The contribution of the latter to the Chow group is at most a finite group [Sch-CM, 5.3]. The assertion follows from the localization sequence (2.2.2).

(3.3) Before describing a field of definition for the cycle class $z_{x}$, it is helpful to introduce the notion of Heegner point in analogy with [Gr]. A point $x \in C M_{D}$ is called a Heegner point if the subgroups $\left\langle s_{1}\right\rangle$ and $\mathscr{H}$ of the three torsion in $\pi^{-1}(x)$ are both $\mathscr{O}_{D}$-submodules. Since the three torsion in $\pi^{-1}(x)$ is isomorphic to $\mathscr{O}_{D} / 3$ as an $\mathscr{O}_{D}$-module, Heegner points only occur when 3 splits in $\mathscr{O}_{D}$. In such cases exactly two points in the $S L(2, \mathbb{Z} / 3) / \pm$ Id-orbit of $x$ are Heegner points. In the analytic picture a point $\tau \in \mathbf{h}$ satisfying an equation with integral coefficients $a \tau^{2}+b \tau+c=0$ with $a>0$ and $\operatorname{gcd}(a, b, c)=1$ is a Heegner point exactly when $3 \mid a$ and $3 \mid c$. Indeed, if $D=b^{2}-4 a c$ then $\mathscr{O}_{D}=Z+Z a \tau=\operatorname{End}(Z+Z \tau)$. So $\mathscr{O}_{D}\langle 1 / 3\rangle=\langle 1 / 3\rangle$ iff $3 \mid a$ and $\mathscr{O}_{D}\langle\tau / 3\rangle=$ $\langle\tau / 3\rangle$ iff $3 \mid c$.

(3.4) Given $D$, the discriminant of an imaginary quadratic order, write $k_{D}=\mathbb{Q}(\sqrt{D}), j\left(\mathscr{O}_{D}\right) \in \mathbb{C}$ for the modular invariant of the lattice, $\mathscr{O}_{D}$, and $H_{D}=k_{D}\left(j\left(\mathscr{O}_{D}\right)\right)$ for the associated ring class field. Given $x \in C M_{D}$ let $H=$ $H_{D}$ if $x$ is a Heegner point and $H=H_{9 D}$ otherwise. By class field theory, $\left(\mathscr{O}_{D} / 3\right)^{*} / \operatorname{im}\left(\mathscr{O}_{D}^{*}\right) \simeq \operatorname{Gal}\left(H_{9 D} / H_{D}\right)$. In particular, $H_{9 D} \simeq H_{D}\left(\mu_{3}\right)$ when $D=1$ $\bmod 3$ or $D=-4 ; \operatorname{Gal}\left(H_{9 D} / H_{D}\right) \simeq \mathbb{Z} / 4$ if $D=-1 \bmod 3$ and $D<-4$; $\operatorname{Gal}\left(H_{9 D} / H_{D}\right) \simeq \mathbb{Z} / 3$ if $D=0 \bmod 3$ and $D<-3$.

Proposition 3.4.1. The point $x$ is defined over $H$.

Proof. By the theory of complex multiplication $[\mathrm{Sh}-3, \S 5] \pi^{-1}(x)$ has a model $E$ defined over $H_{D} \subset H$. If $x$ is a Heegner point, $\left\langle s_{1}\right\rangle$ and $\mathscr{H} \subset E[3]$ are $\operatorname{Gal}\left(\bar{Q} / H_{D}\right)$-modules. After replacing $E$ by its twist with respect to an appropriate quadratic extension of $H_{D}$ if necessary, we find that the Galois group acts trivially on $\left\langle s_{1}\right\rangle$. Thus $s_{1}$ is an $H_{D}$-rational point, and from the Weil pairing, $\mathscr{H} \simeq \mu_{3}$. Now $\left(E, s_{1}, \mathscr{H}\right)$ is an elliptic curve with weak level3-structure defined over $H_{D}$, whence $x \in \dot{X}\left(H_{D}\right)$.

If $x$ is not a Heegner point then one of $\left\langle s_{1}\right\rangle$ or $\mathscr{H}$ is not an $\mathscr{O}_{D}$-module. It is easy to see that the endomorphism ring of $E /\left\langle s_{1}\right\rangle$ or $E / \mathscr{C}$ is $\mathscr{O}_{9 D}$, hence $H_{9 D}$ is contained in any field extension of $H_{D}$ over which the weak level-3structure is defined. A weak level-3-structure on an appropriate twist of $E$ will certainly be defined over the fixed field of the kernel of

$$
\rho: \operatorname{Gal}\left(\bar{Q} / H_{D}\right) \rightarrow \operatorname{Aut}(E[3]) / \operatorname{Aut}(E) .
$$

But $\operatorname{Aut}(E[3]) \simeq\left(\mathscr{O}_{D} / 3\right)^{*}$ and $\operatorname{Aut}(E) \simeq \mathscr{O}_{D}^{*}$. As the fixed field of $\operatorname{Ker}(\rho)$ contains $H_{9 D}$ and $\operatorname{Gal}\left(H_{9 D} / H_{D}\right) \simeq\left(\mathscr{O}_{D} / 3\right)^{*} / \mathscr{O}_{D}^{*}$, these fields are equal.

Corollary 3.4.2. $H$ is the smallest field of definition of the cycle $z_{x}$. 
Proof. Because the formation of $z_{x}$ involves the graph of a complex multiplication, $k_{D}$ is contained in any field of definition. A fundamental theorem [Sh-3, Theorem 5.7] asserts that the smallest extension of $k_{D}$ over which $E$ is defined is $H_{D}$. Observe that the entire Neron-Severi group of the product $E \times E$ is defined over $H_{D}$. The smallest extension of $H_{D}$ over which the weak level-3-structure may be defined is $H$.

Remark 3.4.3. This very simple description of the field of definition of CM points is a happy accident attributable to the fact that the level, $N=3$, satisfies $(\mathbb{Z} / N)^{*} \simeq\{ \pm 1\}$.

(3.5) We close this section by recalling two basic facts about the action of automorphisms on CM cycles. Because the level structure does not enter in the definition of these cycles we have for all $T \in S L(2, \mathbb{Z} / 3)$ that $T_{*} z_{x}=z_{T x}$. For an element $\sigma \in \operatorname{Gal}\left(H_{9 D} / \mathbb{Q}\right)$, it is not difficult to verify that $\sigma\left(z_{x}\right)=\epsilon_{D}(\sigma) z_{\sigma(x)}$, where $\epsilon_{D}$ is the quadratic character associated to the extension $k_{D} / \mathbb{Q}$ [Sch-CM, 2.2].

\section{The action of the Galois group on CM CYCLES}

Fix $D<0$ the discriminant of an imaginary quadratic order. Let $Z(D)$ denote the subgroup of $\bigoplus_{x \in C M_{D}} \mathrm{NS}\left(p^{-1}(x)\right)$ which is generated by complex multiplication cycles. The purpose of this section is to make some progress in the direction of describing the action of the Galois group $\operatorname{Gal}\left(H_{9 D} / \mathbb{Q}\right)$ on the image of $Z(D)$ in $C H^{2}\left(\widetilde{W}_{\mathbb{C}}\right)_{\text {hom }} \otimes \mathbb{Q}$. We are able to proceed far enough to show that the contribution of $\mathrm{CM}$ cycles to $\left(C H^{2}\left(\widetilde{W}_{\mathbb{Q}}\right) \otimes \mathbb{Q}\right)^{\mathrm{Gal}\left(\mathbb{Q} / Q_{0}\right)}$ is zero, where $Q_{0}$ is the compositum of $\mathbb{Q}\left(\mu_{3}\right)$ with all real quadratic fields which are unramified at 3 (cf. 4.4.2). The first step is to pass to a quotient of $Z(D)$ by certain natural equivalences which we now describe.

(4.1) By (2.2.2) the alternating group $A=S L(2, \mathbb{Z} / 3) / \pm 1$ acts on $Z(D)$. The Sylow-2-subgroup $A_{2} \triangleleft A$ acts trivially on the intermediate Jacobian (2.1) and on $C H^{2}\left(\widetilde{W}_{\mathbb{C}}\right)_{\text {hom }} \otimes \mathbb{Q}(2.2)$. For this reason we consider the coinvariants $Z(D)_{A_{2}}$ which form an $\mathbb{Z}\left[A / A_{2}\right] \simeq \mathbb{Z}[\mathbb{Z} / 3]$-module. Given an element, $U \in A$ of order $3,1+U+U^{2}$ annihilates the intermediate Jacobian and $C H^{2}\left(\widetilde{W}_{\mathbb{C}}\right)_{\text {hom }} \otimes$ $\mathbb{Q}$ so we are led to study $\mathscr{Z}(D):=\left(Z(D)_{A_{2}}\right) /\left(1+U+U^{2}\right)$. Since the Sylow-2subgroup of $S L(2, \mathbb{Z} / 3)$ is normal in the semidirect product $\mathscr{G}=S L(2, \mathbb{Z} / 3)$. $\operatorname{Gal}\left(H_{9 D} / \mathbb{Q}\right) \subset \operatorname{Aut}\left(\widetilde{W}_{H_{9 D}}\right)$, we find that $A / A_{2} \cdot \operatorname{Gal}\left(H_{9 D} / \mathbb{Q}\right)$ acts on $Z(D)_{A_{2}}$ which gives rise to an action of $\operatorname{Gal}\left(H_{9 D} / \mathbb{Q}\right)$ on $\mathscr{Z}(D)$.

(4.2) As the set of Heegner points is not preserved by the action of $A_{2}$, it is desirable to work with a slightly larger class of CM points. If $D=1 \bmod 3$ we shall say that a point is special if it is in the $A_{2}$-orbit of a Heegner point. In terms of level structure, this means that the data $\left(E, s_{1}, \mathscr{H}\right)$ gives a special point iff either both $\left\langle s_{1}\right\rangle$ and $\mathscr{H}$ are $\mathscr{O}_{D}$-modules or neither is. When $D \equiv-1$ mod 3 and $D<-4$ it is also of interest to define a notion of special point. In this case $\operatorname{Gal}\left(H_{9 D} / H_{D}\right) \simeq\left(\mathscr{O}_{D} / 3\right)^{*} / \pm 1 \simeq \mu_{8} / \pm 1$, which allows us to identify the element of order two $\sigma \in \operatorname{Gal}\left(H_{9 D} / H_{D}\right)$ with $\sqrt{-1} \in\left(\mathscr{O}_{D} / 3\right)^{*}$. It follows that $\sigma$ acts on the set of four elements (actually the projective line over $\mathbb{Z} / 3$ ), $\mathbb{P} E[3] \simeq \mathbb{P}\left(\mathscr{O}_{D} / 3\right)$, as the product of two disjoint transpositions. The data $\left(E, s_{1}, \mathscr{H}\right)$ is said to correspond to a special point if $\left\langle s_{1}\right\rangle$ and $\mathscr{H} \in \mathbb{P} E[3]$ are interchanged by $\sigma$. Note that $A_{2}$ acts on $\mathbb{P} E[3]$ as the group of all pairs 
of disjoint transpositions, so that the action of $\sigma$ coincides with the action of some element of this commutative group. It follows that the action of $A_{2}$ preserves special points. Here are some other elementary properties of special points valid for $D= \pm 1 \bmod 3, D<-4$.

(4.2.1) The set of special points of discriminant $D, C M_{D}$ sp, is stable under the action of $\operatorname{Gal}\left(H_{9 D} / \mathbb{Q}\right)$.

(4.2.2) $A_{2}$ acts simply transitively on the special points in any $S L(2, \mathbb{Z} / 3)$ orbit $\mathbf{0} \subset C M_{D}$.

(4.2.3) Write $Z_{\mathrm{sp}}(\mathbf{0}) \otimes \mathbb{C} \subset Z(D) \otimes \mathbb{C}$ for the vector space generated by $\mathrm{CM}$ cycles over the special points in $\mathbf{0}$. Then $Z_{\mathrm{sp}}(\mathbf{0})_{A_{2}} \otimes \mathbb{C} \simeq \mathbb{C}$.

Only the first assertion deserves comment. When $D=-1 \bmod 3$ this follows from the fact that $\sigma$ lies in the center of $\operatorname{Gal}\left(H_{9 D} / \mathbb{Q}\right)$. Indeed, $\langle\sigma\rangle$ is a normal subgroup since the fixed field $H_{D}\left(\mu_{3}\right)$ is a Galois extension of $\mathbb{Q}$. When $D=1 \bmod 3(4.2 .1)$ is a consequence of the fact that elements of $A_{2}$ give automorphisms of $X$ which are defined over $\mathbb{Q}(2.1)$.

(4.2.4) A criteria for when a CM point $\tau \in \mathbf{h}$ is special in terms of the triple of integers $(a, b, c)$ associated to $\tau$ (3.3) is needed only later (7.3).

(4.3) Prior to decomposing $\mathscr{Z}(D) \otimes \mathbb{C}$ as a direct sum of irreducible $\mathbb{C}\left[\mathrm{Gal}\left(H_{9 D} / \mathbb{Q}\right)\right]$-modules, we mention a few general facts about the representation theory of Galois groups of ring class fields of imaginary quadratic fields. The main point is that these groups are generalized dihedral groups. In other words they are semidirect products of an abelian normal subgroup $N$ with $\mathbb{Z} / 2$ where the action of $\mathbb{Z} / 2$ is by inversion [La, p. 134]. It is not hard to see that all one dimensional representations of such groups are characters of order two. The remaining complex irreducible representations are two dimensional. They are obtained by inducing characters $\varsigma: N \rightarrow \mathbb{C}^{*}$ where $\varsigma \neq \varsigma^{-1}$. The restriction of such a representation to $N$ is clearly $\varsigma \oplus \varsigma^{-1}$.

(4.4) We adopt the following notation which is motivated by root number calculations for $L_{k}(\widetilde{W}, s)$ : A quadratic field $k$ is said to be of type -1 if it is imaginary and unramified at 3 or is real and ramified at 3 . Any other quadratic field is of type 1 . Write $\delta(k)$ for the type of $k$.

Proposition 4.4.1. If $D \equiv \pm 1$ mod 3 then $\mathrm{Gal}\left(H_{9 D} / H_{D}\left(\mu_{3}\right)\right)$ acts trivially on $\mathscr{Z}(D)$. Every irreducible two dimensional representation of $\mathrm{Gal}\left(H_{D}\left(\mu_{3}\right) / \mathbb{Q}\right)$ occurs in $\mathscr{Z}(D) \otimes \mathbb{C}$ with multiplicity one. Nontrivial one dimensional representations occur with multiplicity one (respectively zero), depending on whether the quadratic field fixed by the kernel is of type -1 (respectively 1). The trivial representation does not occur.

If $D \equiv 0 \bmod 3$, then every irreducible representation of $\mathrm{Gal}\left(H_{9 D} / \mathbb{Q}\right)$ occurs in $\mathscr{Z}(D) \otimes \mathbb{C}$ with multiplicity one except those which factor through the quotient $\operatorname{Gal}\left(H_{D} / \mathbb{Q}\right)$ which do not occur.

Proof. We treat first the case $D \equiv \pm 1 \bmod 3$. By (3.4) $\mathrm{Gal}\left(H_{9 D} / H_{D}\left(\zeta_{3}\right)\right)$ is trivial unless $D<-4$ and $D \equiv-1 \bmod 3$, in which case the group is $\mathbb{Z} / 2$. The resulting involution changes the symplectic level-3-structure but preserves the isomorphism class of each elliptic curve $\pi^{-1}(x)$. The effect on any complex multiplication cycle is the same as an element of $A_{2}$ (4.2), which of course acts trivially on $Z(D)_{A_{2}}$ and $\mathscr{Z}(D)$.

Let $Z_{\mathrm{sp}}(D) \subset Z(D)$ be the subgroup spanned by cycles over special points. We claim that $\operatorname{Gal}\left(H_{D}\left(\zeta_{3}\right) / H_{D}\right)$ acts trivially on the coinvariants $Z_{\mathrm{sp}}(D)_{A_{2}}$. 
When $D \equiv 1 \bmod 3, Z_{\mathrm{sp}}(D)_{A_{2}}$ is generated by Heegner points which are defined over $H_{D}$. Now suppose $D \equiv-1 \bmod 3, D<-4, x \in C M_{D}$ sp , and $\gamma \in \operatorname{Gal}\left(\mathbb{Q} / H_{D}\right)$ maps to a generator of $\operatorname{Gal}\left(H_{9 D} / H_{D}\right)$. Write $\left(E, s_{1}, \mathscr{H}\right)$ for an elliptic curve defined over $H_{D}$ with a weak level-3-structure defined over $\overline{\mathbb{Q}}$ so that this data corresponds to $x$. We claim that there is an $S \in A_{2}$ such that $\left(E, S s_{1}, S \mathscr{H}\right) \simeq\left(E, \gamma s_{1}, \gamma \mathscr{H}\right)$. From the claim and (3.5) we deduce $S z_{x}=z_{S x}=z_{\gamma x}=\gamma z_{x}$, from which the desired triviality of the action of $\operatorname{Gal}\left(H_{D}\left(\mu_{3}\right) / H_{D}\right)$ follows.

Due to the existence of the inversion isomorphism on $E$ it will suffice to construct $S \in A_{2}$ which has the same effect on the points $\left\langle s_{1}\right\rangle, \mathscr{H} \in \mathbb{P} E[3]$ as $\gamma$. We seek a permutation $S$ of the four points $\mathbb{P} E[3]$ which is the product of two disjoint transpositions and which satisfies $S\left\langle s_{1}\right\rangle=\gamma\left\langle s_{1}\right\rangle, S \mathscr{H}=\gamma \mathscr{H}$. Such an $S$ will exist if we can show that $\gamma$ sends the two points $\left\langle s_{1}\right\rangle, \mathscr{H}$ to the two other points in $\mathbb{P E}[3]$. This may be verified by choosing an isomorphism of $\mathscr{O}_{D}$-modules, $\mathscr{O}_{D} / 3 \simeq E[3]$ which sends $\langle 1\rangle$ to $\left\langle s_{1}\right\rangle$ and $\langle\sqrt{-1}\rangle$ to $\mathscr{H}$. Clearly, such an isomorphism exists iff $x$ is special. Using the identification of (3.4) $\mathrm{Gal}\left(H_{9 D} / H_{D}\right) \simeq\left(\mathscr{O}_{D} / 3\right)^{*} /\left(\mathscr{O}_{D}\right)^{*}$ we see that the $\gamma$ action on $\mathbb{P} E[3]$ may be identified with the action of an eighth root of unity, $\zeta_{8} \in\left(\mathscr{O}_{D} / 3\right)^{*}$ on $\mathbb{P}\left(\mathscr{O}_{D} / 3\right)$. Thus $\gamma$ behaves as required.

Let $\mathscr{J}_{D}$ denote the set of $j$-invariants of fractional ideals for $\mathscr{O}_{D}$. Then $\mathscr{J}_{D} \simeq A_{2} \backslash C M_{D}$ sp (4.2.2). By the theory of complex multiplication $\mathscr{J}_{D}$ is a principal homogeneous space for $\mathrm{Gal}\left(H_{D} / k_{D}\right)$ [Sh-3, Theorem 5.7]. Consequently $Z_{\text {sp }}(D)_{A_{2}} \otimes \mathbb{C}$ is isomorphic to the regular representation of $\mathrm{Gal}\left(H_{D} / k_{D}\right)$ (4.2.3). It follows that when $Z_{\mathrm{sp}}(D)_{A_{2}} \otimes \mathbb{C}$ is viewed as a $\mathrm{Gal}\left(H_{D} / \mathbb{Q}\right)$-module, every two dimensional irreducible representation occurs with multiplicity one (4.3). From each pair of one dimensional representations which have the same restriction to $\mathrm{Gal}\left(H_{D} / k_{D}\right)$ exactly one occurs. In fact I claim that it is always the unique member of the pair whose corresponding field is imaginary quadratic. To see this, note that if the two-rank of $\operatorname{Gal}\left(H_{D} / k_{D}\right)$ is $t$, then $\operatorname{Gal}\left(H_{D} / \mathbb{Q}\right)$ has $2^{t+1}$ distinct characters, half of which do not annihilate complex conjugation, $\tau$. From the formula for an induced character [Se, 3.3 Theorem 12] it is clear that if $\rho$ is an irreducible two dimensional representation of $\operatorname{Gal}\left(H_{D} / \mathbb{Q}\right)$ then $\operatorname{tr}(\rho(\tau))=0$. We may now deduce the claim by evaluating the character of the representation $Z_{\mathrm{sp}}(D)_{A_{2}} \otimes \mathbb{C}$ at $\tau$. This amounts to studying the fixed points of the action of complex conjugation on $A_{2} \backslash C M_{D}$ sp $\simeq \mathscr{J}_{D}$. Real $j$-invariants correspond to 2-torsion in Pic $\mathscr{O}_{D}$. Above each of these $2^{t}$-fixed points complex conjugation acts by -1 on the corresponding one dimensional subspace of $Z_{\mathrm{sp}}(D)_{A_{2}} \otimes \mathbb{C}(3.5)$. It follows easily that the trace of $\tau \in \operatorname{End}\left(Z_{\mathrm{sp}}(D)_{A_{2}} \otimes \mathbb{C}\right)$ is $-2^{t}$. Since $2^{t}$ is the number of one dimensional representations which occur when this representation is decomposed, we find that none of these representations annihilates complex conjugation as claimed.

It remains to deduce from the decomposition of the representation of $\mathrm{Gal}\left(H_{D} / \mathbb{Q}\right)$ on $Z_{\mathrm{sp}}(D)_{A_{2}} \otimes \mathbb{C}$ a decomposition of the $\operatorname{Gal}\left(H_{D}\left(\mu_{3}\right) / \mathbb{Q}\right)$-module $\mathscr{Z}(D) \otimes \mathbb{C}$. For $U \in A$ of order $3, \bigcup_{i \in \mathbb{Z} / 3} U^{i} C M_{D \text { sp }}=C M_{D}$. The obvious map gives rise to isomorphisms of $\mathbb{Q}$-vector spaces:

$$
\begin{aligned}
\mathbb{Q}[U] \otimes_{\mathbb{Z}} Z_{\mathrm{sp}}(D) & \simeq Z(D) \otimes \mathbb{Q}, \\
\mathbb{Q}[U] \otimes_{\mathbb{Z}} Z_{\mathrm{sp}}(D)_{A_{2}} & \simeq Z(D)_{A_{2}} \otimes \mathbb{Q},
\end{aligned}
$$




$$
\mathbb{Q}[U] /\left(U^{2}+U+1\right) \otimes_{\mathbb{Z}} Z_{\mathrm{sp}}(D)_{A_{2}} \simeq \mathscr{Z}(D) \otimes \mathbb{Q} .
$$

The Galois group $\operatorname{Gal}\left(H_{D}\left(\mu_{3}\right) / \mathbb{Q}\right) \simeq \operatorname{Gal}\left(H_{D}\left(\mu_{3}\right) / H_{D}\right) \times \operatorname{Gal}\left(H_{D}\left(\mu_{3}\right) / \mathbb{Q}\left(\mu_{3}\right)\right)$ acts on the right-hand side. A compatible action on the left-hand side is obtained by letting the first factor in the product of Galois groups act on the first factor in the tensor product, while $\operatorname{Gal}\left(H_{D}\left(\mu_{3}\right) / \mathbb{Q}\left(\mu_{3}\right)\right)$ acts as discussed above on $Z_{\mathrm{sp}}(D)_{A_{2}}$. A generator of $\operatorname{Gal}\left(H_{D}\left(\mu_{3}\right) / H_{D}\right)$ sends $U$ to $U^{-1}$ which makes the first factor the regular representation for this group. The case $D= \pm 1$ mod 3, $D<-4$ of the proposition now follows. The case $D=-4$ is not difficult and is left as an exercise.

Now suppose that $D=0 \bmod 3, D<-3$. Then $\mu_{3} \subset H_{D}$. For $x \in C M_{D}$, a generator $\sigma \in \mathrm{Gal}\left(H_{9 D} / H_{D}\right)$ fixes the isomorphism class of the curve $\pi^{-1}(x)$ but changes the symplectic level-3-structure. It follows that $\sigma$ has the same effect on the CM cycle $z_{x}$ as an order 3 element in $S L(2, \mathbb{Z} / 3)$ (3.5). In particular, $\operatorname{Gal}\left(H_{9 D} / H_{D}\right)$ permutes simply transitively the $A_{2}$-orbits within any given $A$-orbit in $C M_{D}$. By the theory of complex multiplication [Sh-3, Theorem 5.7] $\mathrm{Gal}\left(H_{D} / k_{D}\right)$ acts simply transitively on the $A$-orbits in $C M_{D}$. It follows that $Z(D)_{A_{2}} \otimes \mathbb{C}$ is isomorphic to the regular representation of $\mathrm{Gal}\left(H_{9 D} / k_{D}\right)$. Since the effect of a generator $\sigma \in \operatorname{Gal}\left(H_{9 D} / H_{D}\right)$ on $z_{x}, x \in C M_{D}$, is the same as that of an order 3 element in $S L(2, \mathbb{Z} / 3)$ the trivial representation of $\operatorname{Gal}\left(H_{9 D} / H_{D}\right)$ does not occur in $\mathscr{Z}(D) \otimes \mathbb{C}$. Thus irreducible representations of $\mathrm{Gal}\left(H_{9 D} / k_{D}\right)$ which factor through $\mathrm{Gal}\left(H_{D} / k_{D}\right)$ do not contribute to $\mathscr{Z}(D) \otimes \mathbb{C}$. The remaining irreducibles appear with multiplicity at most one, in fact exactly one, since $\operatorname{dim}_{\mathbb{C}}(\mathscr{Z}(D) \otimes \mathbb{C})=(2 / 3) \operatorname{dim}_{\mathbb{C}}\left(\mathbb{C}\left[\mathrm{Gal}\left(H_{9 D} / k_{D}\right)\right]\right)$. In particular, characters of order 2 on $\operatorname{Gal}\left(H_{9 D} / k_{D}\right)$ do not appear in this decomposition. By (4.3) the representation of $\mathrm{Gal}\left(H_{9 D} / \mathbb{Q}\right)$ on $\mathscr{Z}(D) \otimes \mathbb{C}$ is completely determined by the restriction to $\operatorname{Gal}\left(H_{9 D} / k_{D}\right)$. The case $D=0$ mod $3, D<-3$ of the proposition follows immediately. When $D=-3$, $\mathscr{Z}(D) \otimes \mathbb{C} \simeq 0$ and $H_{9 D}=H_{D}=k_{D}$, whence the proposition is valid in this case as well.

Corollary 4.4.2. Let $Q_{0}$ denote the compositum of all quadratic fields of type 1 . Then complex multiplication cycles make no contribution to

$$
\left(C H^{2}\left(\widetilde{W}_{\mathbb{Q}}\right) \otimes \mathbb{Q}\right)^{\mathrm{Gal}\left(\mathbb{Q} / Q_{0}\right)} .
$$

Proof. It suffices to show that for each discriminant $D$ of an imaginary qua dratic order, $(\mathscr{Z}(D) \otimes \mathbb{Q})^{\mathrm{Gal}\left(H_{9 D} / H_{9 D} \cap Q_{0}\right)}=0$. But this is an immediate consequence of (4.4.1).

The next corollary makes explicit that if $x$ is a special point, then the element of the Chow group represented by $z_{x}$ is invariant under a larger group than $\operatorname{Gal}\left(\mathbb{Q} / H_{9 D}\right)$. In this sense, the notion of special point is a generalization of the notion of Heegner point.

Corollary 4.4.3. If $x \in C M_{D}$ sp then $z_{x} \in\left(C H^{2}\left(\widetilde{W}_{\mathbb{Q}}\right) \otimes \mathbb{Q}\right)^{\mathrm{Gal}\left(\mathbb{Q} / H_{D}\right)}$.

(4.5) Let $D_{r}, D_{i} \neq 0$ mod 3 denote discriminants of real (respectively imaginary) quadratic fields. If $\left(D_{r}, D_{i}\right)=1$, then $D=D_{r} D_{i}$ is the discriminant of an imaginary quadratic field. By genus theory $k_{D_{i}} \subset H_{D}$. Write $\epsilon_{D_{i}}$ : $\operatorname{Gal}(\mathbb{Q} / \mathbb{Q}) \rightarrow \pm 1$ for the quadratic character corresponding to $k_{D_{i}} / \mathbb{Q}$. By $(4.4 .1)$ $(\mathscr{Z}(D) \otimes \mathbb{Q})^{\epsilon_{D_{i}}}$ is one dimensional. This means that for fixed $D_{i}$ and variable 
$D_{r}$ one finds infinitely many $(\mathscr{Z}(D))$ which contribute cycles defined over $k_{D_{i}}$. Beilinson and Bloch conjecture that the subspace of $C H^{2}\left(\widetilde{W}_{k_{D_{i}}}\right)_{\text {hom }} \otimes \mathbb{Q}$ generated by these cycles is finite dimensional. Whether or not relations of rational equivalence exist among these cycles is unknown. In $\S 7$ we present numerical evidence that modulo Abel-Jacobi equivalence relations do in fact exist; at least when $D_{i}=-4$.

(4.6) The problem of describing the image of the cycle class map cl: $\mathscr{Z}(D) \otimes$ $\mathbb{Q} \rightarrow C H^{2}\left(\widetilde{W}_{\mathrm{C}}\right)_{\text {hom }} \otimes \mathbb{Q}$ remains difficult. It seems however reasonable to hope that $\mathrm{cl}$ is often injective. It would be interesting to investigate this question in a case where numerical computation indicates that the Abel-Jacobi map is not injective (eg. $D=-688$ ) (cf. (7.5)).

\section{L-SERIES}

In this section we discuss the $L$-series $L_{H_{9 D}}\left(H^{3}(\widetilde{W}), s\right)$ which factors as a product of $L$-series parametrized by irreducible representations, $\xi$, of $\operatorname{Gal}\left(H_{9 D} / \mathbb{Q}\right)$. When $D=1 \bmod 3$, there is a correspondence between those factors where the root number is -1 and representations $\xi$ which appear in the decomposition of the $\operatorname{Gal}\left(H_{9 D} / \mathbb{Q}\right)$-module $\mathscr{Z}(D) \otimes \mathbb{C}$. This is analogous to the situation for Heegner points on Weil curves and has the correct flavour for the conjecture of Beilinson and Bloch. However when the imaginary quadratic field $k_{D}$ is $\mathbb{Q}\left(\mu_{3}\right)$ there are infinite families of ray class fields $H_{9 D}$ and representations $\xi$ for which the $L$-series has root number -1 but there are no corresponding CM cycles. Write $\eta_{\mathbb{Q}}$ for the generic point of $X_{\mathbb{Q}}$. The conjecture of Bloch and Beilinson predicts that the Chow group of degree zero cycles on the generic fiber $p^{-1}\left(\eta_{\mathbb{Q}}\right)(X)$ has infinite rank. Presently however this group is not even known to have positive rank.

Some of the $L$-series computations described below have been done using a more sophisticated approach which works for arbitary new forms [Gr] and [K]. However it is of interest here to treat a broader class of representations $\xi$ than in these references.

(5.1) Recall that the $L$-series of $\widetilde{W}$ over a number field $F$ is defined in terms of a system of continuous representations of the Galois group $G_{F}:=\operatorname{Gal}(\overline{\mathbb{Q}} / F)$,

$$
\rho_{F, l}: G_{F} \rightarrow \operatorname{Aut}\left(H^{3}\left(\widetilde{W}_{\mathbb{Q}}, \mathbb{Q}_{l}\right)\right) .
$$

For primes $\mathbf{p}$ of $F$ which do not divide $3 l$ this representation is unramified since $\widetilde{W}$ has good reduction away from 3 . For such primes the polynomial

$$
P_{\mathbf{p}}(t)=\operatorname{det}\left(\mathbf{I d}-\left.t \cdot \operatorname{Frob}_{\mathbf{p}}^{-1}\right|_{H^{3}\left(\widetilde{W}_{\mathbf{Q}}, \mathbb{Q}_{l}\right)}\right)
$$

is independent of $l$ by Deligne's proof of the Weil conjectures. Write $\rho_{F}^{*}$ for the contragredient representation of $\rho_{F, l}$. Define

$$
L_{F}\left(H^{3}(\widetilde{W}), s\right)=L\left(\rho_{F}^{*}, s\right)=\prod_{(\mathbf{p}, 3)=1} P_{\mathbf{p}}\left(N \mathbf{p}^{-s}\right)^{-1} .
$$

This product converges absolutely for $\operatorname{Re}(s)$ sufficiently large. A more precise definition would include factors corresponding to the primes $\mathbf{p} \mid 3$. As we are only concerned with the order of vanishing at the center of the critical strip we may and will ignore such terms. 
Set $\rho=\rho_{\mathbb{Q}}$. It is formal to verify

$$
L\left(\rho_{F}^{*}, s\right)=L\left(\operatorname{Ind}_{F}^{\mathbb{Q}}\left(\rho^{*}\right), s\right)=L\left(\rho^{*} \otimes \operatorname{Ind}_{F}^{\mathbb{Q}} 1, s\right) .
$$

When $F / \mathbb{Q}$ is Galois, the right-hand side factors

$$
L\left(\rho_{F}^{*}, s\right)=\prod_{\xi} L\left(\rho^{*} \otimes \xi, s\right)^{\operatorname{dim} . \xi},
$$

where the product is over the absolutely irreducible representations of $\mathrm{Gal}(F / \mathbb{Q})$. Given a representation, $V$, of $\operatorname{Gal}(F / \mathbb{Q})$ over an algebraically closed field of characterisitic 0 and an irreducible representation $\xi$ write $V^{\xi}$ for the $\xi$-isotypical component and $\nu_{\xi}(V)$ for the multiplicity with which $\xi$ occurs in $V$. Inspired by Gross [Gr], one is led to state the following refinement of the conjecture of Bloch and Beilinson:

$$
\operatorname{dim}\left(C H^{2}\left(\widetilde{W}_{F}\right)_{\text {hom }} \otimes \mathbb{C}\right)^{\xi}=\operatorname{ord}_{s=2} L\left(\rho^{*} \otimes\left(\operatorname{Ind}_{F}^{\mathbb{Q}} 1\right)^{\xi}, s\right) .
$$

Which may be rephrased as

$$
\nu_{\xi}\left(C H^{2}\left(\widetilde{W}_{F}\right)_{\text {hom }} \otimes \mathbb{C}\right)=\operatorname{ord}_{s=2} L\left(\rho^{*} \otimes \xi, s\right) .
$$

We shall be concerned with the case when $F$ is the ring class field $H_{9 D}$.

(5.2) The analytic continuation for (5.1.1) when $F=\mathbb{Q}$ will follow once the $L$-function has been identified with that of a normalized new form. To this end set $q=\exp (2 \pi i \tau / 3)$, and consider the $q$-expansion $\eta^{8}(\tau)=\sum_{n \geq 1} a_{n} q^{n}$ and the associated Dirichlet series $L\left(\eta^{8}(3 \tau), s\right)=\sum_{n \geq 1} a_{n} n^{-s}$. For each prime $p$ not dividing 3 the trace of the Hecke operator of level $p$ is related to the trace of arithmetic Frobenius by [De]

$$
\operatorname{Tr}\left(\rho\left(\operatorname{Frob}_{p}^{-1}\right)\right)=\operatorname{Tr}\left(\left.T_{p}^{*}\right|_{H^{3,0}}\right) .
$$

As the cohomology group $H^{3,0}$ is one dimensional and generated by $\omega$ (1.5) the right-hand term is the coefficient $a_{p}$ in the $q$-expansion of $\eta^{8}(\tau)$. It follows that $L\left(\eta^{8}(3 \tau), s\right)$ has an euler product expansion which, at least away from $p=3$ agrees with (5.1.1) when $F=\mathbb{Q}$.

It turns out that $L\left(\eta^{8}(3 \tau), s\right)$ coincides with the $L$-series of a Hecke character $\Psi$ of the field $K=\mathbb{Q}\left(\mu_{3}\right)$ defined as follows: Let $I_{\sqrt{-3}}$ denote the ideals of $\mathbb{Z}\left[\zeta_{3}\right]$ prime to $\sqrt{-3}$. Define

$$
\Psi: I_{\sqrt{-3}} \rightarrow K^{*}, \quad \Psi(\mathbf{a})=a^{3},
$$

where $(a)=\mathbf{a}$ and $a \equiv 1 \bmod \sqrt{-3}$. Since $\left|N_{K / \mathbb{Q}}(\sqrt{-3}) \cdot \operatorname{Disc}(K)\right|=9$, $L(\Psi, s)$ is the Mellin transform of a weight 4, normalized new form on $\Gamma_{0}(9)$ [Sh, Lemma 3]. There is only one such, namely $\eta^{8}(3 \tau)$, so we may write

$$
L\left(\eta^{8}(3 \tau), s\right)=L(\Psi, s)=\prod_{\mathbf{p} \neq(\sqrt{-3})}\left(1-\Psi(\mathbf{p}) N \mathbf{p}^{-s}\right)^{-1},
$$

where the product is over primes of $K$.

It is also convenient to express the $L$-series on the right-hand side of (5.1.3) as the $L$-series of a Hecke character. Observe that when $F=H_{9 D}(5.1 .2)$ becomes

$$
L\left(\rho_{H_{9 D}}^{*}, s\right)=\prod_{\kappa} L\left(\rho^{*} \otimes \operatorname{Ind}_{k_{D}}^{\mathbb{Q}} \kappa, s\right) \prod_{\gamma} L\left(\rho^{*} \otimes \gamma\right),
$$


where the first product is taken over all characters $\kappa \neq \kappa^{-1}$ of $\operatorname{Gal}\left(H_{9 D} / k_{D}\right)$ and the second over all characters $\gamma: \operatorname{Gal}\left(H_{9 D} / \mathbb{Q}\right) \rightarrow \mu_{2}$. If we abuse notation and write $\gamma$ and $\kappa$ for the corresponding ideal characters we have the following equalities (up to possible disagreement at finitely many bad eul:r factors which does not change the order of vanishing at $s=2)$ :

(5.2.2) $L\left(\rho^{*} \otimes \gamma, s\right)=L\left(\Psi \cdot \gamma \circ N_{K / \mathbb{Q}}, s\right)$,

(5.2.3) If $K \neq k_{D}$ then $L\left(\rho^{*} \otimes \operatorname{Ind}_{k_{D}}^{\mathbb{Q}} \kappa, s\right)=L\left(\Psi \circ N_{K k_{D} / K} \cdot \kappa \circ N_{K k_{D} / k}, s\right)$,

(5.2.4) When $k_{D}=K, L\left(\rho^{*} \otimes \operatorname{Ind}_{K}^{Q} \kappa, s\right)=L(\Psi \cdot \kappa, s) \cdot L\left(\Psi \cdot \kappa^{-1}, s\right)$.

Remark 5.2.5. In fact there is a dominant rational map defined over $\mathbb{Q}$ from the threefold product of the modular curve $X_{0}(27)$ to $\widetilde{W}$ [Sch3, $\left.\S 10\right]$. Hence the equality $L_{\mathbb{Q}}(\widetilde{W}, s)=L(\Psi, s)$ may also be deduced from the fact that $X_{0}(27)$ is an elliptic curve with complex multiplication.

(5.3) The functional equation and analytic continuation for the Hecke $L$ series (5.2.2), (5.2.3), and (5.2.4) follow from Tate's thesis. To describe the precise form of the functional equation it is convenierit to normalize each Groessencharakter, $\Phi$, which appears on the right-hand side of (5.2.2-5.2.4) by setting $\chi(\mathbf{p})=\Phi(\mathbf{p}) /(N \mathbf{p})^{3 / 2}$. Define

$$
L(\chi, s)=\prod_{\mathbf{p}}\left(1-\chi(\mathbf{p})(N \mathbf{p})^{-s}\right)^{-1}=L(\Phi, s+3 / \ddot{z}) .
$$

Write $2 r$ for the degree of the field $M=K k$ or $K$ for which $\Phi$ is a Hecke character. Let $\mathbf{d}$ denote the absolute different of $M$ and $\mathbf{c}$ the conductor of $\Phi$. Set

$$
L^{\#}(\chi, s)=\left(N_{M / \mathbb{Q}} \mathbf{c d}\right)^{r s / 2}(2 \pi)^{r(s+3 / 2)} \Gamma(s+3 / 2)^{r} L(\chi, s) .
$$

Now there is an analytic continuation and functional equation [Lan, Corollary 1, p. 299],

$$
w(\chi) L^{\#}(\chi, s)=L^{\#}(\bar{\chi}, 1-s) .
$$

For the Hecke characters in question $\chi(\overline{\mathbf{p}})=\bar{\chi}(\mathbf{p})$, whence $L^{\#}(\chi, s)=L^{\#}(\bar{\chi}, s)$ and $w(\chi) \in\{ \pm 1\}$. The computation of $w(\chi)$ proceeds via the idele class character $\chi_{\mathbf{A}}$ associated with $\chi$. Set $w(\Phi)=w(\chi)$.

We recall briefly the tools which are used to compute the root number $w(\Phi)$. Write $M_{\mathrm{A}}^{*}$ for the ideles of $M, S$ for the union of the places where $\chi$ ramifies with all archimedian places, and $M_{\mathbf{A}, S}^{*}=\left\{\alpha \in M_{\mathbf{A}}^{*}: \alpha_{\nu}=1\right.$ for $\left.\nu \in S\right\}$. Write $I(\mathbf{c})$ for the fractional ideals prime to the conductor and define

$$
\phi: M_{\mathbf{A}, S}^{*} \rightarrow M_{\mathbf{A}, S}^{*} / \prod_{\nu \notin S} \mathscr{O}_{M_{\nu}}^{*} \simeq I(\mathbf{c}) \quad \text { by } \phi(\alpha)=\prod_{\mathbf{p}} \mathbf{p}^{\text {ord } \alpha} .
$$

Let

$$
\begin{array}{r}
M_{\mathbf{A}, \hat{S}}^{*}=\left\{\alpha \in M_{\mathbf{A}}^{*}: \alpha_{\nu}=1 \text { if } \nu \notin S \text { and } \alpha_{\nu} \in \mathscr{O}_{M_{\nu}}^{*}\right. \\
\text { with } \left.\alpha_{\nu} \equiv 1 \bmod \mathbf{c}_{\nu} \text { if } \nu \in S_{\text {fin }}\right\} .
\end{array}
$$

Now define $\chi_{\mathbf{A}}: M_{\mathbf{A}, S}^{*} \times M_{\mathbf{A}, \hat{s}}^{*} \rightarrow \mathbb{C}^{*}$ by

$$
\chi_{\mathbf{A}}(\alpha)=\chi \circ \phi \circ \operatorname{pr}_{M_{\mathbf{A}, S}^{*}}(\alpha) \cdot \prod_{\nu \in S_{\infty}}\left(\alpha_{\nu} /\left|\alpha_{\nu}\right|\right)^{-3}
$$


where $\operatorname{pr}_{M_{\mathbf{A}, S}^{*}}: M_{\mathbf{A}}^{*} \rightarrow M_{\mathbf{A}, S}^{*}$ is the obvious projection. If $M^{*}$ is viewed as embedded diagonally in $M_{\mathrm{A}}^{*}$, then $M^{*} \cap\left(M_{\mathrm{A}, S}^{*} \times M_{\mathrm{A}, \hat{S}}^{*}\right)$ is contained in the kernel of $\chi_{\mathbf{A}}$ by the product formula. Since $M_{\mathbf{A}}^{*}=M^{*} \cdot M_{\mathbf{A}, S}^{*} \times M_{\mathbf{A}, \hat{S}}^{*}, \chi_{\mathbf{A}}$ is well defined on $M_{\mathbf{A}}^{*} / M^{*}$. The formulae for the local root numbers is given by

Theorem 5.3.1 (Tate). $\quad w(\chi)=\prod_{\nu} w_{\nu}\left(\chi_{\nu}\right)$ where $\chi_{\nu}$ denotes the restriction of $\chi_{\mathrm{A}}$ to $\left(1,1, \ldots, 1, M_{\nu}^{*}, 1, \ldots, 1\right)$ and

(1) If $\nu$ is finite and $\chi_{\nu}$ is unramified, then $w_{\nu}\left(\chi_{\nu}\right)=\chi_{\nu}\left(\mathbf{d}_{\nu}\right)^{-1}$.

(2) If $\nu$ is finite and $\chi_{\nu}$ is ramified with conductor $\mathbf{c}_{\nu}$, then

$$
w_{\nu}\left(\chi_{\nu}\right)=\left(N \mathbf{c}_{\nu}\right)^{-1 / 2} \chi_{\nu}\left(r^{-1}\right) \sum_{a \in \mathscr{O}_{M_{\nu}}^{*} / 1+\mathbf{c}_{\nu}} \chi_{\nu}(a) \exp (2 \pi i \lambda(\operatorname{tr}(a / r)))
$$

where $\lambda$ is the composition of canonical maps

$$
\lambda: \mathbb{Q}_{p} \rightarrow \mathbb{Q}_{p} / \mathbb{Z}_{p} \rightarrow \mathbb{Q} / \mathbb{Z}
$$

and $r$ generates the ideal $\mathbf{d}_{M_{\nu}} \mathbf{c}_{\nu}$.

(3) If $\nu$ is archimedian, $w_{\nu}\left(\chi_{\nu}\right)=(-i)^{3}$.

The following standard corollary is especially useful.

Corollary 5.3.2. Suppose $\rho$ is of finite order. If $\rho$ and $\chi$ have coprime conductors $\mathbf{c}_{\rho}$ and $\mathbf{c}_{\chi}$, then

$$
w(\chi \cdot \rho)=w(\chi) w(\rho) \chi^{-1}\left(\mathbf{c}_{\rho}\right) \rho^{-1}\left(\mathbf{c}_{\chi}\right) .
$$

These formulae, together with a theorem of Froehlich and Queyrut [F-Q, Theorem 2], provide the necessary tools for computing the desired root numbers. The results are summarized in the following theorem.

Theorem 5.3.3. $w(\Psi)=1$ and $L(\Psi, 2) \neq 0$.

(2) Let $L$ denote the quadratic field corresponding to a nontrivial character $\gamma: G_{\mathbb{Q}} \rightarrow \mu_{2}$. Then $w\left(\Psi \cdot \gamma \circ N_{K / \mathbb{Q}}\right)=\delta(L)$, with notation as in (4.4).

For (3)-(5) assume $k \neq K$ is an imaginary quadratic number field, $L \subset K k$ is the maximal subfield which is unramified at 3 , and that $\kappa$ is a ring class character for $k$.

(3) If $\kappa$ is unramified at $3, w\left(\Psi \circ N_{K k / K} \cdot \kappa \circ N_{K k / K}\right)=\delta(L)$.

(4) If $\kappa$ is tamely ramified at 3, $w\left(\Psi \circ N_{K k / K} \cdot \kappa \circ N_{K k / K}\right)=1$.

(5) If $\kappa$ is wildly ramified at $3, w\left(\Psi \circ N_{K k / K} \cdot \kappa \circ N_{K k / K}\right)=-1$.

In (6)-(7) consider the case of Hecke characters for the field $K$. In particular $\kappa$ is a ring class character for this field.

(6) If $\kappa$ ramifies at 3 , then $\kappa \neq \kappa^{-1}$ and $w(\Psi \cdot \kappa)=-w\left(\Psi \cdot \kappa^{-1}\right)$.

(7) If $\kappa$ is unramified at 3 , then $w(\Psi \cdot \kappa)=\epsilon_{-3}(b) \cdot \kappa^{-1}((\sqrt{-3}))$ where $b \mathbb{Z}\left[\zeta_{3}\right]$ is the conductor of the ring class character $\kappa$ with $b \in \mathbb{Z}$ positive. If $\kappa$ has odd order, then $\kappa((\sqrt{-3}))=1$.

Proof. The first assertion in (1) follows immediately from the formulae for the local root numbers. The nonvanishing of the $L$-series was verified by numerical computation. Such computational techniques are discussed in [Bu-G-Z]. As the remainder of the proof is rather tedious we give details only for (7), which is the most mysterious case from the point of view of conjecture (5.1.3) (cf. 5.4). 
Set $\chi(\mathbf{p})=\Psi(\mathbf{p}) /(N \mathbf{p})^{3 / 2}$. By $(5.3 .2)$

$$
w(\chi \cdot \kappa)=w(\chi) w(\kappa) \chi\left(\mathbf{c}_{\kappa}\right)^{-1} \kappa\left(\mathbf{c}_{\chi}\right)^{-1} .
$$

From (1), $w(\chi)=1$. The theorem of Froehlich and Queyrut [F-Q, Theorem 2] gives $w(\kappa)=1$. The conductor of $\kappa, \mathbf{c}_{\kappa}=\mathbf{c}_{\kappa}$, may be written $b \mathbb{Z}\left[\mu_{3}\right]$ with $b$ a positive rational integer prime to 3 . By direct computation, $\chi\left(\mathbf{c}_{\kappa}\right)=\epsilon_{-3}(b)$. Finally $\mathbf{c}_{\chi}=\mathbf{c}_{\Psi}=(\sqrt{-3})$.

To establish the second assertion in (7) use the fact that ideals of $\mathbb{Z}\left[\mu_{3}\right]$ which are pulled back from $\mathbb{Z}$ are annihilated by $\kappa[\mathrm{F}-\mathrm{Q}, \S 3]$. Thus $\kappa((-3))=1$ and the second assertion follows.

The following corollary, which is simply a combination of (5.3.3) and (4.4.1), describes the relationship between root numbers of $L$-series and ranks of certain cycle groups before passing to rational equivalence. Although this relationship is very far from the conjecture (5.1.3), it suggests that CM cycles may go a long ways toward explaining the vanishing of the $L$-functions.

Given an irreducible representation $\xi$ of $\operatorname{Gal}\left(H_{9 D} / \mathbb{Q}\right), w\left(\rho^{*} \otimes \xi\right)$ denotes the root number of the corresponding $L$-series (5.2.2-5.2.4).

Corollary 5.3.4. Case I: $k_{D} \neq K$.

(1) Suppose $3^{2}$ does not divide $D$. If $w\left(\rho^{*} \otimes \xi\right)=1, \nu_{\xi}(\mathscr{Z}(D))=0$. If $w\left(\rho^{*} \otimes \xi\right)=-1, \nu_{\xi}(\mathscr{Z}(D))=1$.

(2) Suppose $3^{2} \mid D$. If $w\left(\rho^{*} \otimes \xi\right)=1, \nu_{\xi}\left(\mathscr{Z}\left(D / 3^{2 j}\right)\right)=0$ for all $j \geq 0$. If $w\left(\rho^{*} \otimes \xi\right)=-1, \nu_{\xi}\left(\mathscr{Z}\left(D / 3^{2 j}\right)\right)=1$ for exactly one $j \geq 0$.

Case II: $k_{D}=K$.

(3) Suppose $\operatorname{dim}(\xi)=1$. Then the same conclusions as in (1) apply.

(4) Suppose $\xi=\operatorname{Ind}_{K}^{Q} \kappa$ with $\kappa$ ramified at $(\sqrt{-3})$, then $w\left(\rho^{*} \otimes \xi\right)=-1$. Furthermore $\nu_{\xi}\left(\mathscr{Z}\left(D / 3^{2 j}\right)\right)=1$ for exactly one $j \geq 0$.

(5) Suppose $\xi=\operatorname{Ind}_{K}^{\mathbb{Q}} \kappa$ with $\kappa$ unramified at $(\sqrt{-3})$, then $L\left(\rho^{*} \otimes \xi, s\right)$ is the product of two $L$ functions with the same root number which is computed in (5.3.1(7)). Furthermore $\nu_{\xi}(\mathscr{Z}(D))=0$.

Recall from (0.2) and (3.2.1) the subgroup $C H^{2}\left(\widetilde{W}_{F}\right)_{C M} \subset C H^{2}\left(\widetilde{W}_{F}\right)_{\text {hom }}$. Corollary (5.3.4) raises the question for which $D$ and $\xi$ might one expect the relation

$$
\operatorname{rank}\left(C H^{2}\left(\widetilde{W}_{H_{9 D}}\right)_{C M} \otimes \mathbb{C}\right)^{\xi}=\operatorname{ord}_{s=2} L\left(\rho^{*} \otimes \xi, s\right) .
$$

In the case that $\xi$ is the quadratic character corresponding to the real quadratic field of discriminant 172 , the left-hand side is zero by (4.4.1) while the $L$ function vanishes to order at least two (8.1-8.2). However this situation seems exceptional, since it depends on the vanishing of a Fourier coefficient in a modular form of weight 5/2. Experience with Heegner points on elliptic curves leads one to expect that the following two hypotheses will frequently hold:

(5.3.6) $\operatorname{ord}_{s=2} L\left(\rho^{*} \otimes \xi, s\right)=0$ or 1 depending upon the parity restriction

$$
\operatorname{ord}_{s=2} L\left(\rho^{*} \otimes \xi, s\right)=\left(w\left(\rho^{*} \otimes \xi\right)-1\right) / 2 \bmod 2 .
$$

(5.3.7) $\mathscr{Z}(D)^{\xi} \rightarrow\left(C H^{2}\left(\widetilde{W}_{H_{9 D}}\right)_{\mathrm{CM}} \otimes \mathbb{C}\right)^{\xi}$ is injective.

In the cases covered by (1)-(4) in (5.3.4) these hypotheses imply

$$
\operatorname{rank}\left(C H^{2}\left(\widetilde{W}_{H_{9 D}}\right)_{\mathrm{CM}} \otimes \mathbb{C}\right)^{\xi} \geq \operatorname{ord}_{s=2} L\left(\rho^{*} \otimes \xi, s\right) .
$$


There are two difficulties in establishing the opposite inequality. As was mentioned in (4.5), when $\xi$ is the character of an imaginary quadratic field, unramified at 3, CM cycles in fibers with complex multiplication by fields other than $k_{D}$ contribute to $Z^{2}\left(\widetilde{W}_{H_{9 D}}\right)_{\text {hom }}$. This situation is considered in $\S 7$ in the case $\xi$ corresponds to the quadratic character attached to $\mathbb{Q}(\sqrt{-1}) / \mathbb{Q}$. As for the second difficulty, note first that $\xi$ may be viewed as a representation of $\mathrm{Gal}\left(H_{9 n^{2} D} / \mathbb{Q}\right)$ by pullback. If $(3, n)=1, \nu_{\xi}\left(\mathscr{Z}\left(n^{2} D\right) \otimes \mathbb{C}\right)=\nu_{\xi}(\mathscr{Z}(D)) \otimes \mathbb{C}$. One would hope that the images of these two vector spaces in $\left(\mathrm{CH}^{2}\left(\widetilde{W}_{H_{9 D}}\right)_{\mathrm{CM}} \otimes \mathbb{C}\right)^{\xi}$ coincide. If the Hecke operators $T_{l},(l, 3)=1$, act by scalars on $C H^{2}\left(\widetilde{W}_{\mathbb{Q}}\right)_{\mathrm{CM}} \otimes \mathbb{C}$, they will give rise to relations between these images. However the action of $T_{l}$ is not presently understood.

Note finally that there is no Hecke operator of level 3. In light of (5.3.5) this could conceivably explain the peculiar form of (2) and (4) in the corollary.

(5.4) Let $K^{\prime}$ denote the composition of all odd order abelian extensions of $K$ which are unramified above 3. By (4.4.1) $C H^{2}\left(W_{K^{\prime}}\right)_{\mathrm{CM}} \otimes \mathbb{C}=0$. The $L$ function for infinitely many subextensions vanishes (5.3.3(7)). It follows easily that the conjecture of Beilinson and Bloch predicts that the Chow group of zero cycles on the generic fiber $p^{-1}\left(\eta_{K^{\prime}}\right)$ which map to zero in the Albanese is of infinite rank. It would be interesting to find such cycles. In analogy with the case of Heegner points on modular elliptic curves, one might look for such cycles first on modular varieties of higher level. If one can produce appropriate cycle classes here, one is still left with the notoriously difficult problem of producing correspondences between higher dimensional varieties which transfer the cycle classes back to $\widetilde{W}$. In light of (5.2.5) it might be more hopeful to apply this philosophy to $X_{0}(27)^{3}$ rather than directly to $\widetilde{W}$.

\section{THE INTERMEDIATE JACOBIAN}

In this section we describe the intermediate Jacobian of $\widetilde{W}^{\text {an }}$ and the strategy for computing the Abel-Jacobi image of an arbitrary CM cycle.

(6.1) By (1.6) and (2.1), $H_{3}\left(\widetilde{W}^{\text {an }}, \mathbb{Z}\right) /$ torsion is a free, rank one $\mathbb{Z}\left[\mu_{3}\right]$ module. Fix a basis $\xi$, set $\Omega=\int_{\xi} \omega$, and define the intermediate Jacobian $J_{1}\left(\widetilde{W}^{\text {an }}\right)=\mathbb{C} / \Omega \mathbb{Z}\left[\mu_{3}\right]$. The Abel-Jacobi map $\alpha: Z^{2}(\widetilde{W})_{\text {hom }} \rightarrow J_{1}\left(\widetilde{W}^{\text {an }}\right)$ is defined by

$$
\alpha(z)=\int_{\mathscr{E}} \omega \bmod \Omega \mathbb{Z}\left[\mu_{3}\right] \in J_{1}\left(\widetilde{W}^{\text {an }}\right),
$$

where $\mathscr{E}$ is a differentiable 3 -chain bounding $z$. For $\tau \in \mathbf{h}$ a CM point, $z_{\tau}$ the corresponding CM cycle, bounding 3-chains $\mathscr{E}_{\tau}$ have been described [Sch-CM, 2.8]. If $a \tau^{2}+b \tau+c=0$ with $a, b, c \in \mathbb{Z}, \operatorname{gcd}(a, b, c)=1, a>0$, and $r=\operatorname{Re}(\tau), s=\operatorname{Im}(\tau)$, then $\int_{\mathscr{E}_{\tau}} \omega$ is given by the formula [Sch-CM, 3.3] where one takes $Q=\mathrm{Id}$. To put this in a more concrete form give $\Delta:=(0, \infty) \times$ $(0,1) \times(0,1)$ the standard product orientation, and define $\phi_{\tau}: \Delta \rightarrow \mathbf{h} \times \mathbb{C}$ by $\phi_{\tau}\left(t, z_{1}, z_{2}\right)=\left(\tau+i t, z_{1}, z_{2}\right)$. Write $q=\exp (2 \pi i \tau / 3), \eta^{8}(\tau)=\sum_{n \geq 1} a_{n} q^{n}$, and set

$$
\beta(\tau)=-\int_{\Delta} \phi_{\tau}^{*} \omega=\frac{2 \pi i}{3} \int_{0}^{\infty} \eta^{8}(\tau+i t) d(i t)=\sum_{n \geq 1} \frac{a_{n} q^{n}}{n} .
$$


Now [Sch-CM, 3.3] may be rewritten

(6.1.2)

$$
\begin{array}{r}
\alpha\left(z_{\tau}\right)=\epsilon(b) a\left(r e^{2 \pi i / 3} \beta(\tau /(-\tau+1))+\left(|\tau|^{2}-r\right) \beta(\tau)-(r-1) \beta(-1 / \tau)\right) \\
\bmod \Omega \mathbb{Z}\left[\mu_{3}\right],
\end{array}
$$

The fastest way to compute the coefficients $a_{n}$ in the power series for $\eta^{8}(\tau)$, is to exploit the happy accident noted in $(5.2)$ that $\eta^{8}(3 \tau)$ is the cusp form associated to the unique Hecke character of infinity type $(3,0)$ on $\mathbb{Q}\left(\mu_{3}\right)$ with conductor $(\sqrt{-3})$. The following description of the coefficients $a_{n}$ is then immediate.

If $n=0 \bmod 3, a_{n}=0$.

For $p$ prime: If $p=-1 \bmod 3$, then $a_{p}=0$.

If $p=1 \bmod 3$, then $a_{p}=\pi^{3}+\bar{\pi}^{3}$, where $p=\pi \bar{\pi}$ in $\mathbb{Z}\left[\mu_{3}\right]$, and $\pi=$ $1 \bmod (\sqrt{-3})$.

For $n$ composite, $(n, 3)=1$, the coefficient $a_{n}$ is determined by the coefficients $a_{p}$ with $p$ a prime factor of $n$ by the usual formulas for eigenfunctions of the Hecke algebra [Ser, VII.5].

The expression (6.1.2) for the Abel-Jacobi image of a CM cycle is well suited for machine computation. The expression for $\beta(\tau)$ converges for all $\tau$ in the upper half-plane, and the convergence is very rapid as long as $\operatorname{Im}(\tau)$ is not too small.

(6.2) It remains to choose an $\mathbb{Z}\left[\mu_{3}\right]$-basis $\xi$ for $H^{3}\left(\widetilde{W}^{\text {an }}, \mathbb{Z}\right) /$ torsion . Roughly the idea is to use the real points of $\widetilde{W}$. However this manifold is not orientable. We pass to a birationally equivalent variety $\hat{W}$ which is defined over $\mathbb{R}$ (in fact over $\mathbb{Q}$ ), has orientable $\hat{W}(\mathbb{R})$, and has the same $H^{3}$ as $\widetilde{W}$. The construction of $\hat{W}$ proceeds by blowing up certain components of the singular fibers of $p: W \rightarrow X$. To begin, let $l$ denote the $\mathbb{Q}$-rational component of $\pi^{-1}(3)$ and write $m_{1}, m_{2}$ for the components which are interchanged by $\operatorname{Gal}\left(\mathbb{Q}\left(\mu_{3}\right) / \mathbb{Q}\right)$. Write $U \in \operatorname{Aut}\left(W_{\mathbb{Q}\left(\mu_{3}\right)}\right)$ for the restriction of $U_{1} \times U_{1} \in$ $\operatorname{Aut}\left(Y \times Y_{\mathbb{Q}\left(\mu_{3}\right)}\right)(2.1)$. Set $L=l \times l, M=m_{1} \times m_{1}+m_{2} \times m_{2}$, and let $W^{\prime}$ denote the variety obtained by blowing up the following sequence of $\mathbb{Q}$-rational Weil divisors on $W$ in the order indicated: $L, U L+U^{2} L, M, U M+U^{2} M$. Next choose an arbirary sequence of irreducible components in the fiber of $W^{\prime}$ over the point $\infty \in X$ with the property that each singular point in this fiber is contained in at least one element of the sequence. Define $\hat{W}$ to be the variety obtained from $W^{\prime}$ by successively blowing up the Weil divisors in this sequence. A local computation at the singularities of $W$ shows that $\hat{W}$ is nonsingular and that the exceptional set is one dimensional. Since the dualizing sheaf of $W$ is trivial, the same holds for the canonical bundle of $\hat{W}$. Note also that $\hat{W}$ is defined over $\mathbb{Q}$ and that the action of $U$ lifts to $\hat{W}_{\mathbb{Q}\left(\mu_{3}\right)}$.

(6.3) Now $\hat{W}(\mathbb{R})$ is orientable since $\hat{W}$ has trivial canonical bundle. Fix the orientation for which $\int_{\hat{W}(R)} \omega+\bar{\omega}$ is positive and let $w \in H_{3}\left(\hat{W}^{\text {an }}, \mathbb{Z}\right) /$ torsion denote the resulting homology class.

Lemma 6.3.1. $w \mathbb{Z}\left[\mu_{3}\right]=3 H_{3}(\hat{W}, \mathbb{Z}) /$ torsion .

Sketch of proof. It suffices to show that the intersection product $w \cdot U_{*} w=9$. In fact

$$
w \cdot U_{*} w=\left(\operatorname{Disc} \mathbb{Z}\left[\mu_{3}\right] w\right)^{1 / 2}=\left[H_{3}\left(\hat{W}^{\text {an }}, \mathbb{Z}\right) / \text { torsion }: \mathbb{Z}\left[\mu_{3}\right] w\right],
$$


and there is a unique $\mathbb{Z}\left[\mu_{3}\right]$-submodule of index 9. The intersection computation may be done directly, but it is complicated by the fact that the cycles do not meet properly. Alternatively one may express $w \cdot U_{*} w$ in terms of certain integrals which may be numerically evaluated. Indeed, consider the closed 3form $\nu=-\left(\omega+\bar{\omega}+2 U^{*}(\omega+\bar{\omega})\right) / 3 R$, where $R=\int_{w} \omega$. It is not difficult to get a good numerical approximation of the real period $R$ as will be explained in (6.4). Write $\mathscr{D}: H_{3}(\hat{W}) \rightarrow H^{3}(\hat{W})$ for the inverse to Poincare duality. From

$$
\int_{\hat{W}(\mathbb{C})} \nu \wedge \mathscr{D} w=\int_{w} \nu=0 \text { and } \int_{\hat{W}(\mathbb{C})} U^{* 2} \nu \wedge \mathscr{D} U_{*} w=\int_{U_{*} w} U^{* 2} \nu=0
$$

we have $\mathscr{D} w=m \nu$ and $\mathscr{D} U_{*} w=m^{\prime} \nu$ for $m, m^{\prime} \in \mathbb{C}$. From

$$
m^{-1}\left(w \cdot U_{*} w\right)=\int_{\hat{W}(\mathbb{C})} \nu \wedge \mathscr{D} U_{*} w=\int_{U_{*} w} \nu=1
$$

and

$$
\left(m^{\prime}\right)^{-1}\left(U_{*} w \cdot w\right)=\int_{\hat{W}(\mathbb{C})} U^{* 2} \nu \wedge \mathscr{D} w=\int_{w} U^{* 2} \nu=-1
$$

follows $m=m^{\prime}=\left(w \cdot U_{*} w\right)$. Thus

$$
\int_{\hat{W}(\mathbb{C})} \nu \wedge U^{* 2} \nu=\left(w \cdot U_{*} w\right)^{-1} \int_{U_{*} w} \nu=\left(w \cdot U_{*} w\right)^{-1} .
$$

Using the well understood action of $S L(2, \mathbb{Z} / 3)$, the computation of the Petersen inner-product $\int_{\hat{W}(\mathbb{C})} \nu \wedge U^{* 2} \nu$ is reduced to the computation of the integral over the standard fundamental domain for $S L(2, \mathbb{Z})$. Crude upper and lower bounds may be obtained by integrating over the regions $|\operatorname{Re}(\tau)| \leq$ $1 / 2, \operatorname{Im}(\tau) \geq \sqrt{3} / 2$ and $|\operatorname{Re}(\tau)| \leq 1 / 2, \operatorname{Im}(\tau) \geq 1$. These estimates suffice to show that the intersection number is less than 11 and greater than 7 . Since the intersection number is the norm of an ideal in $\mathbb{Z}\left[\mu_{3}\right]$ it must be 9 .

Thus we may and will take $\Omega=R / 3$.

(6.4) We derive a rapidly converging infinite series expression for the real period $R$. The real locus of $\dot{W}^{\text {an }}$ is the fixed locus of the involution induced by $\left(\tau, z_{1}, z_{2}\right) \rightarrow\left(-\bar{\tau}, \bar{z}_{1}, \bar{z}_{2}\right)(1.5(4))$. The union of the lines in the upper half-plane $\operatorname{Re}(\tau)=0,3 / 2$ projects bijectively to $\dot{X}(\mathbb{R})$. The fibers of $Y(\mathbb{R})$ over the first line consist of two circles and over the second line of a single circle. It is now straightfoward that

$$
R=\left(\frac{2 \pi i}{3}\right)\left(-4 \int_{0}^{\infty} \eta^{8}(i t) i d t+\int_{0}^{\infty} \eta^{8}\left(\frac{3}{2}+i t\right) i d t\right) .
$$

To relate the integral over $\operatorname{Re}(\tau)=3 / 2$ to the integral over $\operatorname{Re}(\tau)=0$ use the Hecke operator of level 2 which in fact annihilates $\omega$ since the Fourier coefficient $a_{2}=0$. For any prime $p \neq 3$, the formula for the action of the level $p$ Hecke operator

$$
T_{p}^{*} \omega=\left[\left(\sum_{0 \leq k \leq p-1} \eta^{8}\left(\frac{\tau+3 k}{p}\right) p^{-1}\right)+\eta^{8}(p \tau) p^{3}\right]\left(\frac{2 \pi i}{3}\right) d \tau d z_{1} d z_{2}
$$


is well known and follows from the double coset decomposition [Sch-CM, 1.14]. Applying this when $p=2$ yields the relation,

$$
0=\left[\eta^{8}(\tau / 2)+\eta^{8}((\tau+3) / 2)\right] / 2+8 \eta^{8}(2 \tau),
$$

from which

$$
\int_{0}^{\infty} \eta^{8}(3 / 2+i t) i d t=-5 \int_{0}^{\infty} \eta^{8}(i t) i d t
$$

follows. Finally, the functional equation $\eta^{8}(-1 / \tau)=\tau^{4} \eta^{8}(\tau)$ permits one to write the real period as

$$
R=6 \pi \int_{1}^{\infty}\left(1+u^{2}\right) \eta^{8}(i u) d u
$$

Substituting the $q$-expansion for $\eta^{8}(\tau)$ and integrating term by term gives the desired series which converges rapidly to $R$.

(6.5) It will be helpful to say a word about the action of complex conjugation on the intermediate Jacobian. At this point it is simplest to give an entirely ad hoc treatment. Since $\Omega=R / 3$ is a real number, the involution on $\mathbb{C}$ given by -1 times complex conjugation induces an involution on $J_{1}\left(\widetilde{W}^{\text {an }}\right)$. This is compatible with the action of complex conjugation on $\widetilde{W}^{\text {an }}$ and the cycle class map. Indeed if $z \subset \widetilde{W}^{\text {an }}$ is a nullhomologous 1-cycle with $z=\partial \mathscr{E}$, then the conjugation, $c$, on $\widetilde{W}^{\text {an }}$ yields $z^{c}=-\partial \mathscr{E}^{c}$. The minus sign arises because the orientation of the curve is reversed by conjugation. As $\omega=\overline{\omega \circ c}(1.4(4))$,

$$
\alpha\left(z^{c}\right)=-\int_{\mathscr{C} c} \omega=-\overline{\int_{\mathscr{C}} \omega}=-\overline{\alpha(z)}
$$

\section{Computations}

In this section we describe the construction of a class of nullhomologous cycles defined over $\mathbb{Q}(\sqrt{-1})$ and the computation of their images under the Abel-Jacobi map.

(7.1) Let $d \equiv \pm 1 \bmod 3$ be the discriminant of a real quadratic field. Then $D=-4 d$ is the discriminant of the order $\mathscr{O}_{D}:=\mathbb{Z}[(D+\sqrt{D}) / 2]$ in the imaginary quadratic field $k_{D}=\mathbb{Q}(\sqrt{D})$. By genus theory, $H_{D}:=k_{D}\left(j\left(\mathscr{O}_{D}\right)\right)$ contains the subfield $\mathbb{Q}(\sqrt{-1})$. We associate to each such $d$ a cycle $z_{D} \in$ $\mathscr{Z}(D)^{\mathrm{Gal}\left(H_{9 D} / \mathbb{Q}(\sqrt{-1})\right)}$ as follows:

If $D=1 \bmod 3$, let $\tau_{0} \in \mathbf{h}$ denote a Heegner point in the $S L(2, \mathbb{Z})$-orbit of $(D+\sqrt{D}) / 2$. In the corresponding $S L(2, \mathbb{Z} / 3)$-orbit in $\Gamma(3) \backslash \mathbf{h}$ there are two choices for $\tau_{0}$ (3.3). The corresponding CM cycles are defined over $H_{D}$ (3.4) and have the same class in the $\mathscr{Z}(D)(4.2)$. Define

$$
z_{D}=\sum_{\gamma \in \operatorname{Gal}\left(H_{D} / \mathbb{Q}(\sqrt{-1})\right)} z_{\tau_{0}}^{\gamma} .
$$

If $D=-1 \bmod 3$, let $\tau_{0} \in \mathbf{h}$ denote a special point in the $S L(2, \mathbb{Z})$-orbit of $(D+\sqrt{D}) / 2$. Again the cycle class of $z_{\tau_{0}}$ in $\mathscr{Z}(D)$ is independent of the choice of special point. By the proof of (4.4.1), $\mathrm{Gal}\left(H_{9 D} / H_{D}\right) \simeq \mathbb{Z} / 4$ acts trivially on the image of the special points in $\mathscr{Z}(D)$. We set

$$
z_{D}=\frac{1}{4} \sum_{\gamma \in \operatorname{Gal}\left(H_{9 D} / \mathbb{Q}(\sqrt{-1})\right)} z_{\tau_{0}}^{\gamma} .
$$


(7.2) For computational purposes it is convenient to rewrite the expression for $z_{D}$ in terms of ideal classes in $\mathscr{O}_{D}$ in a manner independent of $D$ mod 3 . Let $P_{1}=\operatorname{Gal}\left(H_{D} / k_{D}(\sqrt{-1})\right), P_{-1}=$ the nontrivial coset of $P_{1}$ in $\mathrm{Gal}\left(H_{D} / k_{D}\right)$, and write $c \in \operatorname{Gal}\left(H_{D} / \mathbb{Q}\right)$ for complex conjugation. Then $\operatorname{Gal}\left(H_{D} / \mathbb{Q}(\sqrt{-1})\right)=$ $P_{1} \cup c P_{-1}$. Identify $\mathrm{Gal}\left(H_{D} / k_{D}\right)$ with $\mathrm{Pic} \mathscr{O}_{D}$ via the Artin isomorphism. A well defined quadratic character $\kappa:$ Pic $\mathscr{O}_{D} \rightarrow \mu_{2}$ is induced by composing the norm map on fractional ideals prime to $2, I_{\mathscr{O}_{D}}(2) \rightarrow I_{\mathbb{Z}}(2)$, with the quadratic Dirichlet character $\epsilon_{-4}: I_{\mathbb{Z}}(2) \rightarrow \mu_{2}$ associated to the extension $\mathbb{Q}(\sqrt{-1}) / \mathbb{Q}$. One has $\kappa^{-1}(u)=P_{u}$. For each $\mathbf{a} \in$ Pic $\mathscr{O}_{D}$ write $z_{\mathbf{a}} \in \mathscr{Z}(D)$ for the class of $z_{\tau}$, where $\tau$ is an arbitrary special point for which the $\mathscr{O}_{D}$-module $\mathbb{Z}+\mathbb{Z} \tau$ is in the class a. For $D= \pm 1$ mod 3 we may now write (3.5), (4.2)

$$
z_{D}=\sum_{\mathbf{a} \in \operatorname{Pic} \mathcal{O}_{D}} \kappa(\mathbf{a}) z_{\mathbf{a}} .
$$

(7.3) The actual procedure for computing the image, $\alpha\left(z_{D}\right)$, of $z_{D}$ under the Abel-Jacobi map will now be described. The first step is to make a list, $T^{\prime}$, of all triples of integers $(a, b, c)$ with $a>0, \operatorname{gcd}(a, b, c)=1, b^{2}-4 a c=$ $D, c \geq a \geq|b|$. If two triples in $T^{\prime}$ have the same values of $a$ and $c$ we discard the triple for which $b<0$. This gives rise to a subset $T^{\prime \prime} \subset T^{\prime}$. By the classical theory of primitive binary quadratic forms of discriminant $D$ [Bo-Sh, §2.7], the set $T=\left\{\tau \in \mathbf{h}: a \tau^{2}+b \tau+c=0\right.$ for $\left.(a, b, c) \in T^{\prime \prime}\right\}$ is in bijective correspondence with Pic $\mathscr{O}_{D}$ via $\tau \rightarrow \mathbb{Z}+\mathbb{Z} \tau$. Furthermore each $\tau \in T$ lies in the standard fundamental domain for the action of $S L(2, \mathbb{Z})$ on $\mathbf{h}$. To each $\tau \in T$ there is a element $R_{\tau} \in S L(2, \mathbb{Z} / 3)$, uniquely determined up to the Sylow-2-subgroup, such that $R_{\tau} \tau$ is a special point. Let $r_{\tau} \in \mu_{3}$ denote the unique scalar satisfying $R_{\tau}^{*} \omega=r_{\tau} \omega$.

Lemma 7.3.1. Let $\zeta_{3} \in \mathbf{h}$ be the cube root of 1 . The formula for $r_{\tau}$ is

(1) When $D=-1 \bmod 3, r_{\tau}=1$ iff $b=0 \bmod 3$. If $b \neq 0 \bmod 3$, then $a \neq 0 \bmod 3$ and $r_{\tau}=\zeta_{3}^{-b / a \bmod 3}$.

(2) When $D=1 \bmod 3, r_{\tau}=1$ if both $a$ and $c$ or neither $a$ nor $c$ is divisible by 3. If $3 \mid a$ but not $c, r_{\tau}=\zeta_{3}^{b / c \bmod 3}$ and if $3 \mid c$ but not $a$, $r_{\tau}=\zeta_{3}^{-b / a \bmod 3}$.

Proof. Left to the reader (cf. (3.3)).

It remains to evaluate the quadratic character $\kappa$.

Lemma 7.3.2. For $\tau \in T$ we may take $\kappa(\mathbb{Z}+\mathbb{Z} \tau)=\epsilon_{-4}(a)$ if $a$ is odd and $\kappa(\mathbb{Z}+\mathbb{Z} \tau)=\epsilon_{-4}(c)$ if $a$ is even.

Proof. The lattice $\mathbb{Z}+\mathbb{Z} \tau$ is a fractional ideal for the order $\mathscr{O}_{D}=\mathbb{Z}+\mathbb{Z} a \tau$. The reciprocal of the norm of $\mathbb{Z}+\mathbb{Z} \tau$ is $|(\mathbb{Z}+\mathbb{Z} \tau) /(\mathbb{Z}+\mathbb{Z} a \tau)|=a$. If $a$ is odd, the formula for $\kappa(\mathbb{Z}+\mathbb{Z} \tau)$ follows. If $a$ is even, then $D=b^{2}-4 a c=0$ mod 4 , implies $b$ is even, whence $c$ is odd, by $\operatorname{gcd}(a, b, c)=1$. Now the lattice $\mathbb{Z}+\mathbb{Z}(-1 / \tau)$ corresponds to the same $\mathscr{O}_{D}$-module as $\mathbb{Z}+\mathbb{Z} \tau$ and has $(c,-b, a)$ as associated 3-tuple. The formula for $\kappa(\mathbb{Z}+\mathbb{Z} \tau)$ when $a$ is even follows.

Thus the Abel-Jacobi image

$$
\alpha\left(z_{D}\right)=\sum_{\tau \in T} \kappa(\mathbb{Z}+\mathbb{Z} \tau) r_{\tau} \alpha\left(z_{\tau}\right)
$$


is presented in a form suitable for machine computation.

(7.4) It remains to derive from the numerical value $\alpha\left(z_{D}\right) \bmod \Omega \mathbb{Z}\left[\mu_{3}\right]$ a convincing argument that $\alpha\left(z_{D}\right)$ is an integral multiple of a fixed reference point $j \in J_{1}\left(\widetilde{W}^{\text {an }}\right)$ for all $D$. For $j$ we choose the Abel-Jacobi image of the cycle $z_{\sqrt{-1}}$. The Galois group $\operatorname{Gal}(\mathbb{Q} / \mathbb{Q})$ operates on the class of this cycle in $\mathscr{Z}(-4)$ via the quadratic character for $\mathbb{Q}(\sqrt{-1}) / \mathbb{Q}$. Similary in $Z_{\mathrm{sp}}(D)_{A_{2}}$ complex conjugation acts by $c\left(z_{D}\right)=-z_{D}$, since $\kappa(\mathbf{a}) c\left(z_{\mathbf{a}}\right)=-\kappa(\mathbf{a}) z_{c \cdot \mathbf{a}}=-\kappa(c \cdot \mathbf{a}) z_{c \cdot \mathbf{a}}$. As the Abel-Jacobi map on complex multiplication cycles of discriminant $D$ factors through $\mathscr{Z}(D),(6.5)$ implies that $\alpha\left(z_{\sqrt{-1}}\right)$ and $\alpha\left(z_{D}\right)$ are contained in $\mathbb{R} / \Omega \mathbb{Z} \subset J_{1}\left(\widetilde{W}^{\text {an }}\right)$.

The procedure used to decide if $\alpha\left(z_{D}\right)$ is indeed an integral multiple of $\alpha\left(z_{\sqrt{-1}}\right)$ is as follows. Write $\tilde{\alpha}\left(z_{\tau}\right)$ for the complex number on the right-hand side of (6.1.2) which represents $\alpha\left(z_{\tau}\right) \in J_{1}\left(\widetilde{W}^{\text {an }}\right)$ and define

$$
\tilde{\alpha}\left(z_{D}\right)=\sum_{\tau \in T} \kappa(\mathbb{Z}+\mathbb{Z} \tau) r_{\tau} \tilde{\alpha}\left(z_{\tau}\right) .
$$

Solve $\tilde{\alpha}\left(z_{D}\right)=\tilde{\alpha}_{1}\left(z_{D}\right)+\tilde{\alpha}_{2}\left(z_{D}\right) \zeta_{3}$ for $\tilde{\alpha}_{1}\left(z_{D}\right), \quad \tilde{\alpha}_{2}\left(z_{D}\right) \in \mathbb{R}$. Note that in fact $\tilde{\alpha}_{2}\left(z_{D}\right) \in \mathbb{Z}$. These numbers were evaluated numerically using the computer language interactive basic on an IBM personal computer. The infinite series (6.1.1) was approximated by the first 193 terms. Six significant digit accuracy was expected and indeed $\tilde{\alpha}_{2}\left(z_{D}\right)$ was always found to be an integer up to this accuracy. For each integer $m$ of absolute value less than $70+\left|\tilde{\alpha}_{1}\left(z_{D}\right)\right|$ the computer determined if $\left(\tilde{\alpha}_{1}\left(z_{D}\right)+m \Omega\right) / \tilde{\alpha}_{1}\left(z_{\sqrt{-1}}\right)$ is within $10^{-4}$ of an integer. For each $D$ tested there was precisely one $m$ for which this condition was satisfied. The associated integers, $c_{d}$ where $D=-4 d$ and $d<250$ is the discriminant of a real quadratic number field, are tabulated below. This provides some evidence in favour of the conjecture of Bloch and Beilinson. Additional evidence is provided by the fact that the $c_{d}$ 's are essentially the Fourier coefficients of a particular weight $5 / 2$ cusp form as will be discussed in the next section.

(7.5) TABLE. $\alpha\left(z_{D}\right)$ as a multiple of $\alpha\left(z_{\sqrt{-1}}\right)$.

\begin{tabular}{|rrrrrr|}
\hline \multicolumn{3}{|c}{$D=-4 d=1$} & $\bmod 3$ & \multicolumn{3}{c|}{$D=-4 d=-1 \bmod 3$} \\
$d$ & $D$ & $c_{d}$ & $d$ & $D$ & $c_{d}$ \\
5 & -20 & -3 & 13 & -52 & 3 \\
8 & -32 & 6 & 28 & -112 & 12 \\
17 & -68 & -3 & 37 & -148 & -12 \\
29 & -116 & 9 & 40 & -160 & -6 \\
41 & -164 & 15 & 61 & -244 & 6 \\
44 & -176 & -24 & 73 & -292 & -15 \\
53 & -212 & 3 & 76 & -304 & -24 \\
56 & -224 & 12 & 85 & -340 & 27 \\
65 & -260 & -24 & 88 & -352 & -12 \\
77 & -308 & -18 & 97 & -388 & 21 \\
89 & -356 & 15 & 109 & -436 & -27 \\
92 & -368 & 24 & 124 & -496 & 12 \\
101 & -404 & -15 & 133 & -532 & -6 \\
104 & -416 & -24 & 136 & -544 & 30 \\
\hline
\end{tabular}


(7.5) TABLE (continued)

\begin{tabular}{|rrrrrr|}
\hline \multicolumn{2}{r}{$D=-4 d=1$} & \multicolumn{3}{c|}{$D=-4 d=-1 \bmod 3$} \\
$d$ & $D$ & $c_{d}$ & $d$ & $D$ & $c_{d}$ \\
113 & -452 & -27 & 145 & -580 & 3 \\
137 & -548 & 63 & 157 & 628 & -6 \\
140 & -560 & 24 & 172 & -688 & 0 \\
149 & -596 & 69 & 181 & -724 & 9 \\
152 & -608 & -48 & 184 & -736 & 60 \\
161 & -644 & -42 & 193 & -772 & 24 \\
173 & -692 & 9 & 205 & -820 & 15 \\
185 & -740 & -6 & 217 & -868 & 24 \\
188 & -752 & 24 & 220 & -880 & -48 \\
197 & -788 & -33 & 229 & -916 & -27 \\
209 & -836 & -6 & 232 & -928 & -54 \\
221 & -884 & -42 & 241 & -964 & 21 \\
233 & -932 & 57 & & & \\
236 & -944 & 48 & & & \\
248 & -992 & 36 & & & \\
\hline
\end{tabular}

\section{A MODULAR FORM OF WEIGHT $5 / 2$}

In this section we show that the integers $c_{d}$ in (7.5) are, up to a fixed constant multiple depending only on $d$ mod 3 , precisely the Fourier coefficients of a cusp form of weight $5 / 2$ on $\Gamma_{0}(36)$. The idea that this might be the case is due to Dick Gross. We also discuss briefly where this idea comes from.

(8.1) The relationship between forms of half-integral weight and forms of even weight was discovered by Shimura. An introduction to these ideas may be found in [Ko-1, IV]. Given an odd integer $k \geq 5, g \in S_{k / 2}\left(\Gamma_{0}(4 N)\right)$ an eigenform for all Hecke operators $T_{p},(p, 4 N)=1$, with corresponding eigenvalues $\lambda_{p}$, Shimura [Sh-2] constructs a weight $k-1$ cusp form $f$ satisfying $\left.f\right|_{T_{p}}=\lambda_{p} f$. It turns out that $f \in S_{k-1}\left(\Gamma_{0}(2 N)\right)$ [Ni]. In the case of interest here $f(\tau)=\eta^{8}(3 \tau) \in S_{3}\left(\Gamma_{0}(9)\right)$ and Koblitz [Ko-2, p. 216] gives the following description of a corresponding $g \in S_{5 / 2}\left(\Gamma_{0}(36)\right)$ : Let $g_{1}(\tau)$ denote the unique weight two normalized new form on $\Gamma_{0}(36)$, and write $\theta(\tau)=\sum_{n \in \mathbf{Z}} q^{n^{2}}$, where $q=\exp (2 \pi i \tau)$ for the classical theta function. Then $g=\sum_{n \geq 1} b_{n} q^{n}:=\left.\left(g_{1} \theta\right)\right|_{1+(1 / 4) T_{4}}=\left.\left(\sum_{n \geq 1} b_{n}^{\prime} q^{n}\right)\right|_{1+(1 / 4) T_{4}}$. The Fourier coefficients are quickly computed since $b_{n}=b_{n}^{\prime}+(1 / 4) b_{4 n}^{\prime}$, and $g_{1}$ is associated to the unique Hecke character on $\mathbb{Q}\left(\mu_{3}\right)$ of infinity type $(1,0)$ with conductor $(2 \sqrt{-3})$. As noted in the introduction, for $d<250, d \equiv \pm 1 \bmod 3$ the discriminant of a real quadratic field, we find only one $b_{d}$ which vanishes, namely $b_{172}$. In fact for all $d$ in this range we find the relationship $b_{d}=-\epsilon_{-3}(d) 2 c_{d}$, where $\epsilon_{D}$ denotes the Dirichlet character of the quadratic field of discriminant D.

(8.2) The importance of the Fourier coefficients $b_{d}$ where $d$ is the discriminant of a real quadratic field lies in their relationship to the central critical values of twists of the $L$-function $L(f, s)=\sum_{n \geq 1} a_{n} n^{-s}$. For $d$ prime to the level of $f$ define $L\left(f, \epsilon_{d}, s\right)=\sum_{n \geq 1} \epsilon_{d}(n) a_{n} n^{-s}$. Now a deep result of Waldspurger [Wa, Corollary 2] applied to the particular case at hand implies 
that there is a constant $C_{1} \neq 0$ depending only on $d$ mod 3 such that

$$
d^{3 / 2} L\left(f, \epsilon_{d}, 2\right)=C_{1} b_{d}^{2} \text {. }
$$

A consequence is that the vanishing of the twisted $L$-function is equivalent to $b_{d}=0$.

(8.3) It remains to speculate about the relationship between the central critical values of twists of the $L$-function $L\left(\eta^{8}(3 \tau), s\right)$ and Abel-Jacobi images of algebraic cycles. We begin by describing briefly the analogous situation with Heegner points on modular elliptic curves, where much is known thanks to the work of Gross and Zagier [G-Z]. We recall some of their results in a somewhat specialized context. Fix a positive integer $N$ which for simplicity we assume to be the level of a genus 1 modular curve $X_{0}(N)$. Choose odd integers $D=d_{1} d_{2}$ all of which are discriminants of quadratic fields and such that $k_{D}$ and $k_{d_{2}}$ are imaginary. Further assume $(D, N)=1$ and that all primes which divide $N$ split in $k_{D}$ and $k_{d_{2}}$. This last assumption guarantees the existance of Heegner points of level $N$ and discriminants $D$ and $d_{2}$ [Gr, §3]. The quadratic character on the ideles of $k_{D}, \chi=\epsilon_{d_{i}} \circ N_{k_{D} / \mathbb{Q}}$, corresponds to the field extension $k_{D} k_{d_{i}} / k_{D}$ and is independent of $i \in\{1,2\}$. Write $f(\tau)=\sum_{n \geq 1} a_{n} \exp (2 \pi i n \tau)$ for the normalized new form on $\Gamma_{0}(N)$ of weight 2 and set

$$
L\left(f, \epsilon_{d_{i}}, s\right)=\sum_{n \geq 1} \epsilon_{d_{i}}(n) a_{n} n^{-s}, \quad L(f, \chi, s)=L\left(f, \epsilon_{d_{1}}, s\right) \cdot L\left(f, \epsilon_{d_{2}}, s\right) .
$$

The root numbers in the functional equations for $L(f, s), L\left(f, \epsilon_{d_{2}}, s\right)$, $L\left(f, \epsilon_{d_{1}}, s\right)$, and $L(f, \chi, s)$ are respectively +1 (since $X_{0}(N)$ has genus $1[\mathrm{Li}]), \epsilon_{d_{2}}(-N)=-1, \epsilon_{d_{1}}(-N)=1$ [We, Satz 1], and -1 .

For $x \in X_{0}(N)\left(H_{D}\right)$ a Heegner point of discriminant $D$, Gross and Zagier consider the divisor $c_{\chi}=\sum_{\sigma \in \operatorname{Gal}\left(H_{D} / k_{D}\right)} \chi\left(\sigma^{-1}\right)(x)^{\sigma}$, which up to sign is independent of the choice of Heegner point. This divisor class is evidently defined over $k_{D} k_{d_{2}}$. The generator of $\operatorname{Gal}\left(k_{D} k_{d_{2}} / k_{D}\right)$ acts by multiplication by -1 . Using the fact that $w_{N}$ acts by inversion on $\mathrm{Pic}^{0}\left(X_{0}(N)\right)$, one can also check that complex conjugation $\delta$ acts on $c_{\chi}$ by multiplication by $-\epsilon_{d_{1}}(N)=-1$ [G-Z, II.1], [Bi-S]. Thus $c_{\chi}$ is defined over $k_{d_{2}}$. Now Gross and Zagier prove [G-Z, I.6, IV.0.4]

$$
|D|^{1 / 2} L^{\prime}(f, \chi, 1)=C_{0} \hat{h}_{\mathbb{Q}}\left(c_{\chi}\right),
$$

where $\hat{h}_{\mathbb{Q}}$ denotes the canonical height and $C_{0}$ is a constant independent of $D$ and $\chi$. This equation may be rewritten as

$$
d_{1}^{1 / 2} L\left(d, \epsilon_{d_{1}}, 1\right)=\left[\left|d_{2}\right|^{1 / 2} L^{\prime}\left(f, \epsilon_{d_{2}}, 1\right) / C_{0}\right]^{-1} \hat{h}_{\mathbb{Q}}\left(c_{\chi}\right)
$$

For fixed $d_{2}$ but variable $d_{1}$ the term in square brackets is constant.

(8.4) We now speculate as to how (8.3.2) might generalize to the situation at hand. Beilinson [Be] and Bloch [B14] have sought to generalize the height pairing to nullhomologous 1-cycles on threefolds. In the case of CM cycles additional progress has been made recently by Brylinsky [Br]. Take $f(\tau)=\eta^{8}(3 \tau), d_{2}=$ $-4, d_{1}=d= \pm 1 \bmod 3$, the discriminant of a real quadratic field. The root numbers for $L(f, s), L\left(f, \epsilon_{-4}, s\right), L\left(f, \epsilon_{d}, s\right)$ are $1,-1,1$ respectively. In analogy with (8.3.2) one might hope for a formula

$$
4^{3 / 2} L^{\prime}\left(f, \epsilon_{-4}, 2\right) d^{3 / 2} L\left(f, \epsilon_{d}, 2\right)=C_{0} \hat{h}\left(z_{D}\right)
$$


where $\hat{h}$ is a height function and $C_{0}$ is a constant depending only on $d$ mod 3 . (When $D=-4 d=1 \bmod 3, z_{D}$ is constructed from Heegner cycles, otherwise from slightly more general $\mathrm{CM}$ cycles.) Although we have only given evidence that $z_{D}$ and $c_{d} z_{\sqrt{-1}}$ are Abel-Jacobi equivalent, it is tempting (given our total ignorance of the actual truth!) to hope that they might also have the same height. In this case the right-hand side of $(8.4 .1)$ could be replaced by $C_{0} c_{d}^{2} \hat{h}\left(z_{\sqrt{-1}}\right)$, yielding (8.2.1)

$$
c_{d}^{2}=C d^{3 / 2} L\left(f, \epsilon_{d}, 2\right)=C^{\prime} b_{d}^{2}
$$

for constants $C$ and $C^{\prime}$ depending only on $d$ mod 3 . The relationship which is actually observed $b_{d}=-\epsilon_{-3}(d) 2 c_{d}$ (8.1) is consistent with these speculations and is in fact slightly stronger in that the predicted relationship seems to actually hold between $b_{d}$ and $c_{d}$ rather than just between the squares.

The question as to whether or not the cycles $z_{D}$ and $b_{d} z_{\sqrt{-1}}$ have the same class in $C H^{2}\left(\widetilde{W}_{\mathbb{Q}(\sqrt{-1})}\right) \otimes \mathbb{Q}$ remains wide open. Hopefully further developements in the theory of heights for nullhomologous cycles of higher codimension will lead to additional insight. A purely geometric investigation could also be interesting. In any case we note that an analogous result for Heegner cycles on modular curves has been proved [G-K-Z] and [Za].

ivote added in proof. J. Nekovár reports progress on a $p$-adic analog of the conjectured formula (8.4.1).

\section{REFERENCES}

[AMRT] A. Ash, D. Mumford, M. Rapoport, and Y. Tai, Smooth compactification of locally symmetric varieties, Math. Sci. Press, Brookline, Mass., 1975.

[Be] A. Beilinson, Height pairing between algebraic cycles, Contemp. Math., vol. 67, Amer. Math. Soc., Providence, R.I., 1987.

[Bi] L. Bianchi, Ueber die Normalformen dritter und fuenfter Stufe des elliptischen Integrals erster Gattung, Math. Ann. 17 (1880), 234-268.

[Bi-S] B. Birch, and N. Stephens, Computation of Heegner points, Modular Forms, (R. A. Rankin, ed.), Wiley, New York, 1984.

[Bl] S. Bloch, Algebraic cycles and values of L-functions, J. Reine Angew. Math. 350 (1984), 94-108.

[B12] _ Algebraic cycles and values of L-functions. II, Duke Math. J. 52 (1985), 379-397.

[B13] Z Zero cycles on abelian surfaces, Appenidx to: Zero cycles on surfaces with $p_{g}=0$, Compositio Math. 33 (1976), 135-145.

[B14] _- Height pairings for algebraic cycles, J. Pure Appl. Algebra 34 (1984), 119-145.

[B15] _ Lectures on algebraic cycles, Duke Univ. Math. Series, IV, Durham, N.C., 1980.

[Bl-S] S. Bloch and V. Srinivas, Remarks on correspondences and algebraic cycles, Amer. J. Math. 105 (1983), 1235-1253.

[Bo-Sh] Z. Borevitch and I. Shafarevich, Number theory, Academic Press, New York, 1966.

[Br] J.-L. Brylinski, Heights for local systems on curves, Duke Math. J. 59 (1989), 1-26.

[Bu-G-Z] J. Buhler, B. Gross, and D. Zagier, On the conjecture of Birch and Swinnerton-Dyer for an elliptic curve of rank 3, Math. Comp. 44 (1985), 473-481.

[De] P. Deligne, Formes modulaires et representations l-adiques, Séminaire Bourbaki 355, Lecture Notes in Math., vol. 179, Springer, New York, 1971, pp. 139-172.

[F-Q] A. Froehlich, and J. Queyrut, On the functional equation of the Artin L-function for characters of real representations, Invent. Math. 20 (1973), 125-138. 
[G-Z] B. Gross and D. Zagier, Heegner points and derivatives of L-series, Invent. Math. 84 (1986), 225-320.

[G-K-Z] B. Gross, W. Kohnen and D. Zagier, Heegner points and derivatives of L-series. II, Math. Ann. 278 (1987), 496-562.

[Gr] B. Gross, Heegner points on $X_{0}(N)$, Modular Forms, (R. A. Rankin, ed.), Wiley, New York, 1984.

[H] R. Hartshorne, Algebraic geometry, Graduate Texts in Math. 52, Springer, New York, 1977.

[Ha] B. Harris, Homological versus algebraic equivalence in a Jacobian, Proc. Nat. Acad. Sci. U.S.A. 80 (1983), 1157-1158.

[Ko-1] N. Koblitz, Introduction to elliptic curves and modular forms, Graduate Texts in Math. 97, Springer, New York, 1984.

[Ko-2] N. Koblitz, p-adic congruences and modular forms of half integral weight, Math. Ann. 274 (1986), 199-220.

[Ku] P. K. Kurcanov, The zeta function of elliptic curves over certain abelian extensions of imaginary quadratic fields, Math. Sb. (N.S) 102(144) (1977), 56-70.

[La] S. Lang, Elliptic functions, Addison-Wesley, Reading, Mass, 1973.

[Lan] _ Algebraic number theory, Addison-Wesley, Reading, Mass., 1970.

[Li] G. Ligozat, Courbes modulaires de genre 1, Bull. Soc. Math. France, Mem. 43, Supplement au Bull. Soc. Math. France 103 (1975).

[M-S] D. Mumford, and K. Suominen, Introduction to the theory of moduli, Algebraic Geometry, (Oslo 1970), (F. Oort, ed.), Wolters-Noordhoff, Groningen, 1972, pp. 171-222.

[Mu] J. Murre, Un resultat en theorie des cycles algebriques de codimension deux, C.R. Acad. Sci. Paris Ser. I 296 (1983), 981-984.

[Ni] S. Niwa, Modular forms of half-integral weight and the integral of certain theta functions, Nagoya Math. J. 56 (1975), 147-161.

[Sch-CM] C. Schoen, Complex multiplication cycles on ellipitc modular threefolds, Duke Math. J. 53 (1986), 771-794.

[Sch-FP] C. Schoen, Fiber products of rational elliptic surfaces with section, Math. Z. 197 (1988), 177-199.

[Sch3] __ On the computation of the cycle class map for nullhomologous cycles over the algebraic closure of a finite field, manuscript (1989).

[Se] J. P. Serre, Linear representations of finite groups, Graduate Texts in Math., vol. 42, Springer, New York, 1977.

[Ser] _ _ A course in arithmetic, Graduate Texts in Math., vol. 7, Springer, New York, 1973.

[Sh-1] G. Shimura, On elliptic curves with complex multiplication as factors of the Jacobians of modular function fields, Nagoya Math. J. 43 (1971), 199-208.

[Sh-2] G. Shimura, On modular forms of half-integral weight, Ann. of Math. 97 (1973), 440-481.

[Sh-3] G. Shimura, Introduction to the arithmetic theory of automorphic functions, Princeton Univ. Press, 1971.

[Shd] T. Shioda, On elliptic modular surfaces, J. Math. Soc. Japan 24 (1972), 20-59.

[Top] J. Top, Hecke L-series related with algebraic cycles or with Siegel modular forms, Thesis, Univ. of Utrecht, 1989.

[Wa] J. L. Waldspurger, Sur les coefficients de Fourier des formes modulaires de poids demi-entier, J. Math. Pures Appl. 60 (1981), 375-484.

[We] A. Weil, Ueber die Bestimmung Dirichletscher Reihen durch Funktionalgleichungen, Math. Ann. 168 (1967), 149-156.

[Za] D. Zagier, Modular points, modular curves, modular surfaces and modular forms, Lecture Notes in Math., vol. 1111, Springer, 1985, pp. 225-248.

[Ze] D. Zelinski, Some abelian threefolds with nontrivial Griffiths group, Compositio Math. 78 (1991), 315-355.

Department of Mathematics, Duke University, Durham, North Carolina 27706

E-mail address: schoen@math.duke.edu 